ENERGY PARTITIONING, ENERGY COUPLING (EPEC) EXPERIMENTS AT THE NATIONAL IGNITION FACILITY

K. B. Fournier, C. G. Brown , M. J. May, W. H. Dunlop, S. M. Compton, J. O. Kane, P. B. Mirkarimi, R. L. Guyton, E. Huffman

January 13, 2012 
This document was prepared as an account of work sponsored by an agency of the United States government. Neither the United States government nor Lawrence Livermore National Security, LLC, nor any of their employees makes any warranty, expressed or implied, or assumes any legal liability or responsibility for the accuracy, completeness, or usefulness of any information, apparatus, product, or process disclosed, or represents that its use would not infringe privately owned rights. Reference herein to any specific commercial product, process, or service by trade name, trademark, manufacturer, or otherwise does not necessarily constitute or imply its endorsement, recommendation, or favoring by the United States government or Lawrence Livermore National Security, LLC. The views and opinions of authors expressed herein do not necessarily state or reflect those of the United States government or Lawrence Livermore National Security, LLC, and shall not be used for advertising or product endorsement purposes.

This work performed under the auspices of the U.S. Department of Energy by Lawrence Livermore National Laboratory under Contract DE-AC52-07NA27344. 


\title{
ENERGY PARTITIONING, ENERGY COUPLING (EPEC) EXPERIMENTS AT THE NATIONAL IGNITION FACILITY
}

\author{
Kevin B. Fournier, Charles G. Brown Jr., Mark J. May, William H. Dunlop, \\ Steven M. Compton, Jave O. Kane, and Paul B. Mirkarimi \\ Lawrence Livermore National Laboratory
}

Robert L. Guyton and Eric Huffman

National Securities Technologies

\begin{abstract}
The energy-partitioning, energy-coupling (EPEC) experiments at the National Ignition Facility (NIF) will simultaneously measure the coupling of energy into both ground shock and air-blast overpressure from a laser-driven target. The source target for the experiment is positioned at a known height above the ground-surface simulant and is heated by four beams from NIF. The resulting target energy density and specific energy are equal to those of a low-yield nuclear device. The ground-shock stress waves and atmospheric overpressure waveforms that result in our test system are hydrodynamically scaled analogs of seismic and air-blast phenomena caused by a nuclear weapon. In what follows, we discuss the motivation for our investigation and briefly describe NIF. Then, we introduce the EPEC experiments, including diagnostics, in more detail.
\end{abstract}

\section{Introduction}

For consequence management, knowing the yield of a nuclear detonation is important in informing the population of the affected area about necessary shelter-in-place or evacuation measures in the immediate aftermath of the explosion. The relationship between the masses of fissile material in a device, the yield, and the measured radiochemistry data from the device debris was well established during the US nuclear test program. This can be simply understood as follows:

$$
\text { Mass of material / (atoms/fission) } \propto \text { Fission yield }
$$

Where atoms/fission is a fundamental radiochemical quantity determined by measuring the fission products in a debris sample and the number of atoms of a specific isotope in that same debris sample. This is rearranged to be 
Number of fissions $\times$ atoms/fission $\propto$ Mass of material

or

Yield $\times$ atoms/fission $\propto$ Mass of material

Since measuring the yield of nuclear test explosions has been done using seismic and other means for decades it might seem that the measurement of the yield of a low-yield nuclear explosion would be straightforward. However, the measurement of yields of explosions done as part of a nuclear testing program were generally done when weather conditions were favorable and the placement of the test device, whether underground or above the surface, were chosen to facilitate a good measurement of the yield.

Accurately measuring the yield of a nuclear detonation in an unknown placement in a structured environment is a much more challenging problem. For any nuclear detonation near the surface of the earth, most of the energy is expressed in a combination of ground shock and air blast. For explosions slightly under the surface, the majority of the energy will be in the ground shock while for an explosion slightly above the surface, most of the energy will be in the air blast. But the sum of these two components of the energy should be, to first order, independent of the placement of the device.

The US entered into a moratorium on nuclear testing in 1992 and signed the Comprehensive Test Ban Treaty (CTBT) in 1996. (However, the US has yet to ratify the CTBT.) Thus, there is at the present time no capability to generate new data on the effects of a nuclear explosion in a structured environment. However, the development of high-powered lasers to support Inertial Confinement Fusion (ICF) and experiments to support the Stockpile Stewardship Program provide the capability to pursue new approaches to generating data to support the investigation of nuclear weapons effects in structured environments.

The concept of scaled experiments is not new. In "The Effects of Nuclear Weapons" page 101-102, Glasstone and Dolan [1] identifies the basic scaling laws for nuclear weapons (see also Appendix A):

Theoretically, a given pressure will occur at a distance from an explosion that is proportional to the cube root of the energy yield. Full-scale tests have shown this relationship between distance and energy yield to hold for yields up to (and including) the megaton range. Thus, cube root scaling may be applied with confidence over a wide range of explosion energies. 
For explosions of different energies having the same scaled height of burst, the cube root scaling law may be applied to distances from ground zero, as well as to distances from the explosion. Thus, if $d_{1}$, is the distance from ground zero at which a particular overpressure or dynamic pressure occurs for a 1-kiloton explosion, then for an explosion of $W$ kilotons energy the same pressures will be observed at a distance $d$ determined by the relationship

$$
d=d_{1} \times W^{1 / 3}
$$

Cube root scaling can also be applied to arrival time of the shock front, positive phase duration, and positive phase impulse, with the understanding that the distances concerned are themselves scaled according to the cube root law.

High explosive (HE) tests have been performed on scaled systems using the cuberoot scaling as presented in Glasstone and Dolan. Several test series including the Cowboy series and Magdeliana Mine experiments that evaluated the capability to decouple nuclear explosions in underground cavities were conducted as scaled experiments using HE. In these experiments the energy in the explosive charge is scaled. The distances and times are then appropriately scaled by the cube root of the ratio of the scaled mass to the actual mass of $\mathrm{HE}$. For such scaled HE testing, the scaling of the distance and time is usually in the range of 10 to 100 while the scaling of the $\mathrm{HE}$ mass is scaled by a factor of $10^{3}$ to $10^{6}$. All of these scaled experiments rely on the similarity conditions of the Euler equations [2] that predict the hydrodynamic behavior of a system undergoing a strong shock $[3,4]$. The EPEC experiment satisfies the conditions for the strong drive case. (The physics of the hydrodynamically scaled EPEC system are laid out in Appendix A.)

The basic approach of the EPEC experiment is to direct several kilojoules of laser energy into a halfraum. A halfraum is a variation of the hohlraums that are widely used in laser experiments [5]. A hohlraum generally has two entrance holes for the laser energy to enter the target, whereas a halfraum only has one laser entrance hole. The majority of the laser energy is converted into radiation inside of the halfraum and within a few nanoseconds the radiation energy is converted to kinetic energy of the halfraum walls with some fraction of the energy radiating from the halfraum walls. Some small fraction of the laser energy is scattered back out through the laser entrance hole and is lost from the experiment. The heated halfraum results in a system that has a plasma core that is radiating energy in the x-ray spectral range. In this sense it is similar to a nuclear device where the energy is deposited by fission and the resulting plasma radiates in the $x$-ray regime. More details on the EPEC halfraum and the full EPEC system are given in Sections IV.A and IV.B, respectively. 


\section{Simulations}

One may understandably be skeptical of the scaling of phenomena across 9 orders of magnitude ( $2.5 \mathrm{kT} \approx 10^{13} \mathrm{~J}$ of weapon energy to $10^{4} \mathrm{~J}$ of laser energy). We have used numerical simulations to evaluate the hydrodynamic response of earth-like materials in our laboratory system to high-energy-density blast sources and to model the energy coupling/partitioning curve for low-yield nuclear detonations close to the Earth's surface. A mathematical derivation of the direct correspondence of the hydrodynamic evolution of two scaled systems is given in Appendix A. Using LLNL's supercomputers and the GEODYN hydrodynamic code [6,7], the properties of stress, pressure, and energy were evaluated for simulated $2.5 \mathrm{kT}$ detonations at various heights above the surface and depths below the surface. ${ }^{1}$ The results indicate stronger air blasts for detonations above or near the surface and that energy coupling into the ground changes rapidly with detonation location over a very small range between the above ground and below-ground interface. This work has set the baseline for designing our NIF experiments.

The hydrodynamic code GEODYN [6,7] was developed at LLNL and incorporates physical models to describe fully a broad range of phenomena including shock and thermodynamic behavior. The code satisfies the thermodynamic conditions and Euler equations discussed in Appendix A. GEODYN is an Eulerian code, which means the mesh or background is stationary and the material is allowed to move though stationary cells, with adaptive mesh refinement (AMR) [8]. The adaptive mesh means that the code has the ability to vary the level of detail of the background. An Eulerian code with AMR such as GEODYN allows for rigorous high numerical resolution in areas in one part of a problem and less refined, less sensitive areas in another. Details on the system of equations solved by GEODYN are given in Appendix $A$.

Figure 1 shows a comparison of two GEODYN runs for two 'air blast' high-energydensity events. One run (right) is for a $2.5 \mathrm{kT}$ simulated nuclear device over an infinite plane of limestone and desert air, the other (left) is for a simulated $10.5 \mathrm{~kJ}$ NIF experiment. Many of the qualitative features are very similar in the two runs, such as the spherical expansion of the blast wave and the formation of a Mach stem at the airground interface in both problems. Further, the absolute scales for pressure and velocity (color scales) are the same in the two runs, which shows that those quantities are invariant between the two systems. This is a consequence of the hydrodynamic scaling of the spatial and temporal dimensions; spatial and temporal scales in the simulations differ by $10^{3}$, the energy source in the two problems differ by $10^{9}$.

\footnotetext{
${ }^{1}$ Nearly all these initial simulations were done by Dr. Otis Walton of LLNL, and separately by US Army Captain Brian Holloway as part of his Master's thesis.
} 

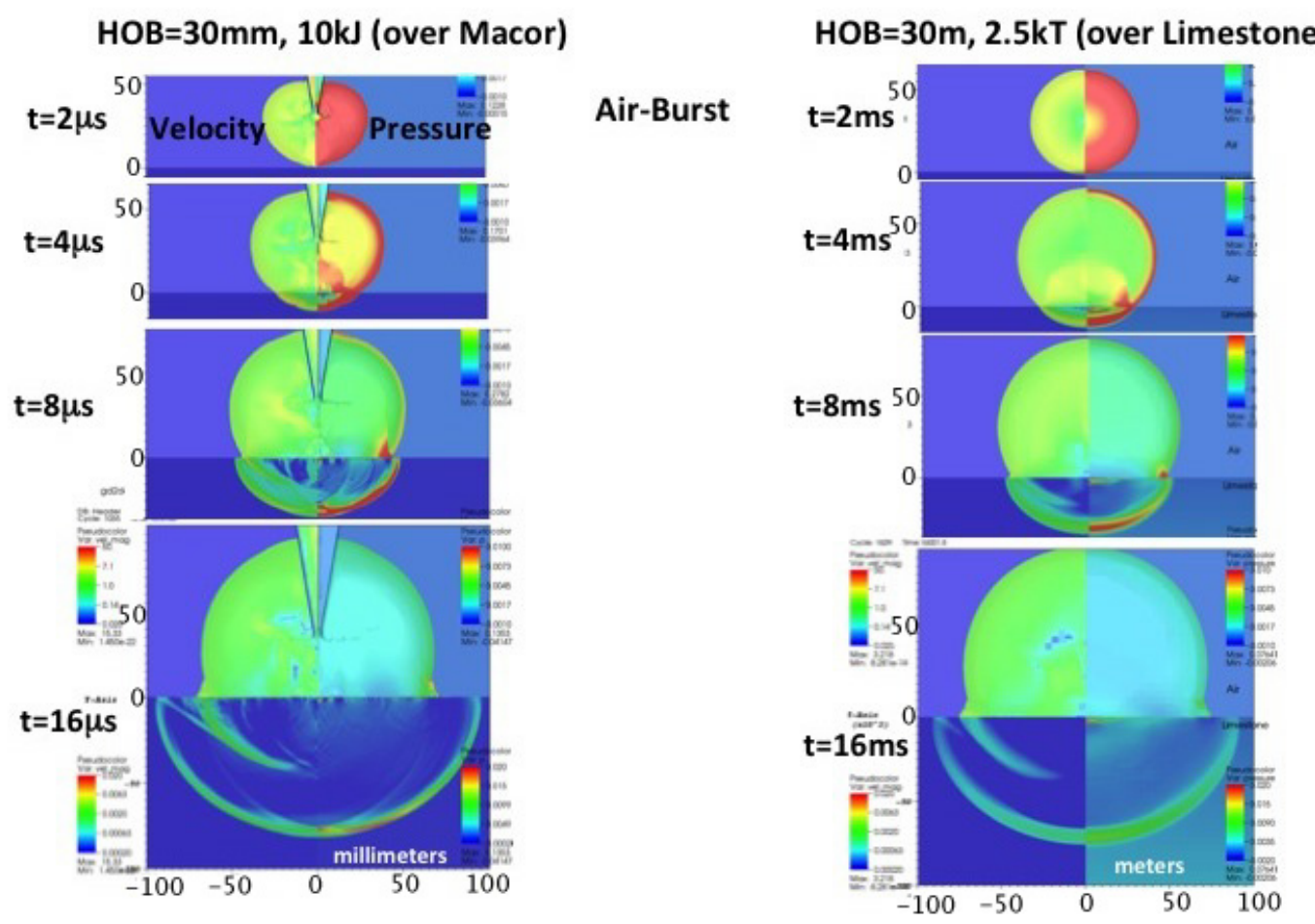

Figure 1 - (left) simulation of a $10.5 \mathrm{~kJ}$ NIF experiment with the laser target $30 \mathrm{~mm}$ above the ground surface and (right) a $2.5 \mathrm{kT}$ nuclear blast $30 \mathrm{~m}$ above the ground surface. One can see in the NIF simulation a cone entering from the top of the picture through which the laser propagates to the target. Color maps in each set of images are for blast velocities (left side) and pressure fields (right side). The color scales in both the NIF and nuclear simulations are on the same absolute scale. The time of each image is written next to the frame (microseconds for the NIF experiment, milliseconds for the nuclear event), the spatial scales are indicated along the edge of each frame (millimeters for the NIF experiment, meters for the nuclear event).

Figure 2 shows GEODYN simulations of the type discussed in the paragraph above for three emplacement scenarios: air blast, surface blast and shallowly buried. Again, both qualitatively and quantitatively, the simulated phenomena in each case are strikingly similar between the $2.5 \mathrm{kT}$ full-scale and $10 \mathrm{~kJ}$ NIF-scale calculations. The shallowly buried high-energy-density event is the one with the greatest differences between the two cases. The reason is that the laser-entrance cone that is a necessity of the NIF experiment provides relief for the blast in the ground simulant that is absent in the full-scale case. 


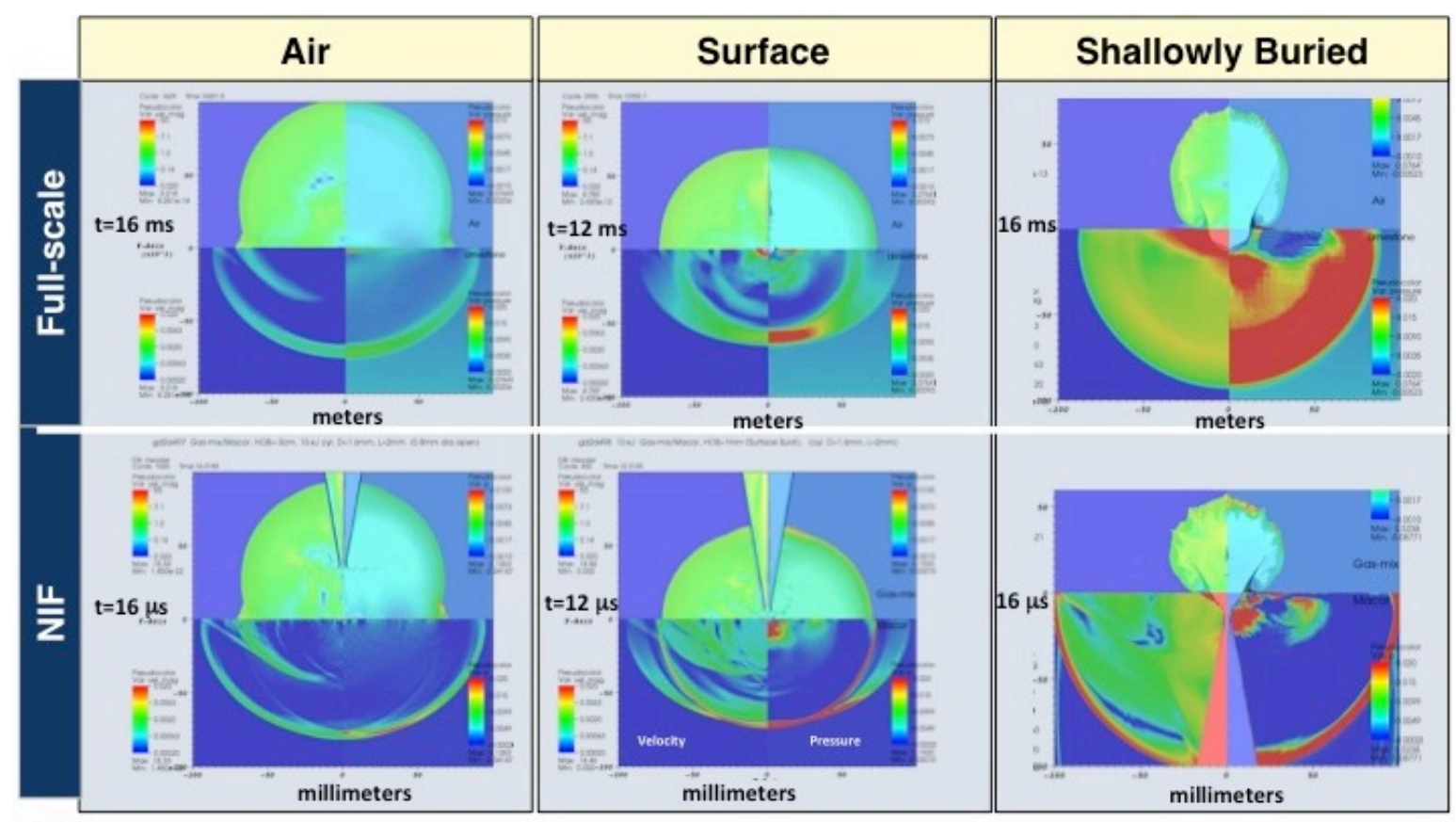

Figure 2 - Snapshots of simulations for (left) air blast, (center) surface blast and (right) shallowly buried high-energy-density events. The simulations are (top) for a 2.5 kT nuclear weapon (i.e., full scale) and (bottom) for a $10.5 \mathrm{~kJ}$ (i.e., NIF scale) shot. Spatial and temporal scales differ by $10^{3}$ between the full- and NIF-scale simulations; times are indicated next to each frame (milliseconds for full scale, microseconds for NIF scale); spatial scales are the same as in Fig. 1: from -100 to $+100 \mathrm{~m}$ for full scale, -100 to $+100 \mathrm{~mm}$ for NIF scale.; color maps are the same in the two sets of simulations. 


\section{The National Ignition Facility}

An excellent introduction to NIF can be found in Ref. 9, from which this introductory material is taken. (See also Ref. 10 for technical details.) NIF, with 192 beams and megajoule capability, is the highest-energy laser in the world. NIF laser pulses begin as low-energy pulses that are split into 48 beams for preamplification. The 48 preamplified beams are then divided into 4 beams each, a "quad", for further amplification before entering the target chamber. The whole laser system can deliver $1.8 \mathrm{MJ}$ of ultraviolet $(351 \mathrm{~nm})$ laser light to a target in the target chamber. NIF laser pulses can range up to 20 ns in duration, and individual quads can be given delays relative to the other quads. For EPEC, only one quad of the available 48 is used to deliver $10.5 \mathrm{~kJ}$ of energy in a $1 \mathrm{~ns}$ pulse.

The NIF target chamber is $10 \mathrm{~m}$ in diameter and can be accessed by three diagnostic instrument manipulators (DIMs). The DIMs are the interfaces by which diagnostic systems can be configured and inserted into the vacuum of the NIF target chamber. The DIMs allow for highly accurate positioning of diagnostic systems (on the order of 100's of microns or better) relative to target-chamber center. Systems in the DIMs are constrained by the 12 " aperture of the gate valve that forms the barrier between the DIM workspace and the target chamber vacuum. It is worth noting that the EPEC experiment described here clears this limiting aperture by only $20 \mathrm{~mm}$.

\section{Energy-partitioning, Energy-coupling Experiments}

As stated in the introduction, the purpose of the EPEC experiments is to measure ground-shock and air-blast overpressure created by a laser-driven target, which is positioned at a known height of burst with respect to the ground-surface simulant. This platform will provide data from a well-controlled set of conditions that will be used for validation of codes. This experimental program is to be accomplished in two phases: The first, an Energetics Campaign, will serve to qualify the target and measure key quantities necessary for success in the following phase, the Data Campaign that will produce the actual data for code-validation exercises. In the following subsections we describe the EPEC experiments, and the diagnostics, in terms of the two phases.

\section{IV.A. Energetics Campaign}

In our Energetics Campaign, we will characterize the energy balance in the EPEC halfraum target. We will measure the laser-target coupling, that is, the laser energy reflected from and absorbed by the halfraum target will be measured. We will also measure the laser-to-x-ray conversion efficiency of the deposited energy; the time history of $x$-ray flux in the halfraum target as well as the spectral and spatial distribution of $x$ rays that are emitted through the halfraum wall will be measured. Following the 
deposition of approximately $10 \mathrm{~kJ}$ of laser energy, the radiation field inside the halfraum target is at $100-200 \mathrm{eV}$ in temperature ( $1 \mathrm{eV}=11604.5 \mathrm{~K}$ ).

\section{IV.A.1. Target Fabrication and Metrology}

The target is a hollow silver sphere (Figure 3) produced by General Atomics ${ }^{2}$. The target is $2 \mathrm{~mm}$ in diameter, and has a 7 or $15 \mu \mathrm{m}$ thick wall. The total mass of the ball is $0.93 \mathrm{mg}$ of silver for the $7 \mu \mathrm{m}$ thick wall. The laser entrance hole (LEH) is $800 \mu \mathrm{m}$ in diameter, and is visible in the wire-mesh rendering in Fig. 3. There is a silver, conical skirt around the LEH. The conical section is part of the structural support to maintain the vacuum channel for the laser beam entering the spherical halfraum for the full Data Campaign experiment. The halfraum is fabricated by plating silver onto a diamondturned aluminum mandrel to a thickness greater than that which is desired. The silver is then back machined to the specified thickness, which presents a great challenge in the precision required in the machining of silver to the desired $7 \mu \mathrm{m}$ thickness with $1 \mu \mathrm{m}$ uniformity. The aluminum mandrel is then is subsequently removed. Final determination of the wall thickness is made by use of $\mathrm{x}$-ray opacity information obtained with an XRadia ${ }^{\mathrm{TM}}$ system. [11] Accurately characterizing these thin hollow spheres by nondestructive techniques such as XRadia ${ }^{\mathrm{TM}}$ has been very challenging, both in terms of assessing the wall thickness and residual aluminum contamination. Additionally, we have employed a destructive characterization technique using a dual focused ion beam (FIB) and scanning electron microscope (SEM) tool on selected targets to benchmark the XRadia ${ }^{\mathrm{TM}}$ results. An image of the FIB section of an out of specification EPEC part is shown in Fig. 3. [12] In the figure, both the silver wall thickness and a layer of residual aluminum are visible. Future targets will be made on plastic mandrels, which will remove the issue of the residual Al contamination.

${ }^{2}$ General Atomics in La Jolla, CA, see http://www.ga.com/index.php for company details. 
The left-hand image in Fig. 3 is made using a state-of-the-art optical measuring system to measure coordinates on the assembled targets. In order to locate the laser entrance hole of the target to a $\pm 100 \mu \mathrm{m}$ accuracy in the NIF target chamber, a small alignment fiber with characteristic $\approx 100 \mu \mathrm{m}$ diameter drops of glue is attached to each target and the relative positions of the glue drops with respect to the LEH are measured

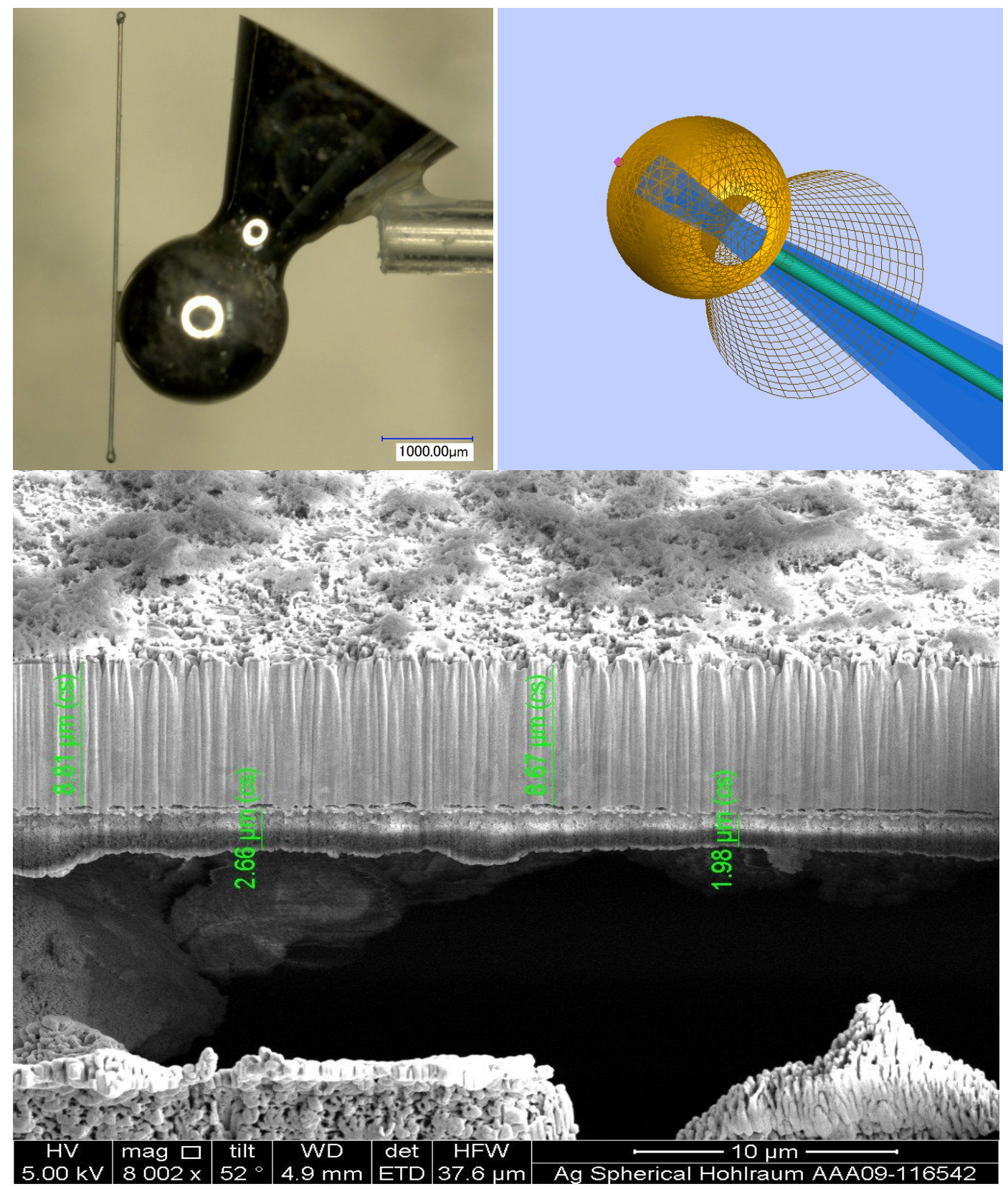

Figure 3 - (upper left) Photo of EPEC target EPEC_A_A_01 with alignment fiber attached, and (upper right) wire mesh rendering of target showing the NIF laser cone entering the target. The target is a spherical silver halfraum, with the spherical part having a diameter of $2 \mathrm{~mm}$. (lower) A FIB cut into the wall of a silver spherical halfraum, the silver thickness is observed to be approximately $8.7 \mu \mathrm{m}$, while a $\approx 2 \mu \mathrm{m}$ layer of what is apparently aluminum lines the inner surface of the sphere. 
for each as-built target using the optical coordinate measuring system. These glue fiducials are then used to position the LEH precisely at the center of the target chamber, which is where the NIF laser beams are aimed. It is interesting to note that a small amount of laser energy, as well as x-ray flux from the halfraum interior, strikes the edges of the laser entrance hole during the experiment causing it to expand and close in about approximately 2 ns.

As explained below, during the later Data Campaign, the spherical EPEC halfraum will have the NIF target chamber vacuum in its interior, and will hold off $1 \mathrm{~atm}$ of pressure on its exterior. We have done tests using a vacuum system to measure the significant pressure differential, up to $2 \mathrm{~atm}$, across the thin walled spheres that are needed for the final EPEC experiments. Both our $15 \mu \mathrm{m}$ and $7 \mu \mathrm{m}$ wall targets survived intact, giving us confidence looking ahead to the Data Campaign. [12]

\section{IV.A.2. Measured Energetics Data}

The key parameters to measure in our Energetics Campaign are the laser-to-target coupling, the conversion of laser to x-ray energy, and the time history and total x-ray flux that emerges through the target wall for the two wall thicknesses. The laser-totarget coupling is determined by measuring how much of the laser power is reflected or scattered (via interactions between the laser field and plasma instabilities [13]) during the pulse and lost through the LEH. Comparing the integral of the scattered laser power to the total laser energy delivered to the target defines the coupling. The laser energy not lost via scattering is then available to interact with and be absorbed by the target. The absorbed laser energy goes into ablating material from the target surface, ionizing target atoms, and accelerating electrons in low-density regions of the target plasma. The absorbed energy is then partitioned between stored internal energy in the resulting plasma ions, kinetic energy of the target debris and both continuum and discrete line radiation from the hot plasma components. It is the $\mathrm{x}$-ray radiation from the hot target plasma that drives blast, shock and fireball phenomena in our full EPEC system, which is why we want to quantify the target's x-ray output during our Energetics Campaign.

The spherical EPEC halfraum with its approximately $10 \mathrm{~kJ}$ of absorbed laser energy is the scaled source for the EPEC experiment. This source has the same energy per unit mass and volume as a low yield nuclear weapon. The EPEC experiments were designed using a scale factor of $1 / 1000$ for the linear dimension. This corresponds to a volume scale of $1 / 10^{9}$. Thus, the 1 to 2 milligram mass of the halfraum target is equivalent to 1000 to $2000 \mathrm{~kg}$ in the full-scale system and the proposed $10 \mathrm{~kJ}$ energy in the halfraum corresponds to $10^{10} \mathrm{~kJ}$ (2.5 kilotons) in the full-scale system. The $1 \mathrm{~mm}$ radius of the hohlraum corresponds to a radius of 1 meter in the full-scale system. Thus the physical extent, the energy per mass and the energy per volume are representative of the scaled size, mass, and energy density of a low-yield (few kiloton) nuclear device. 


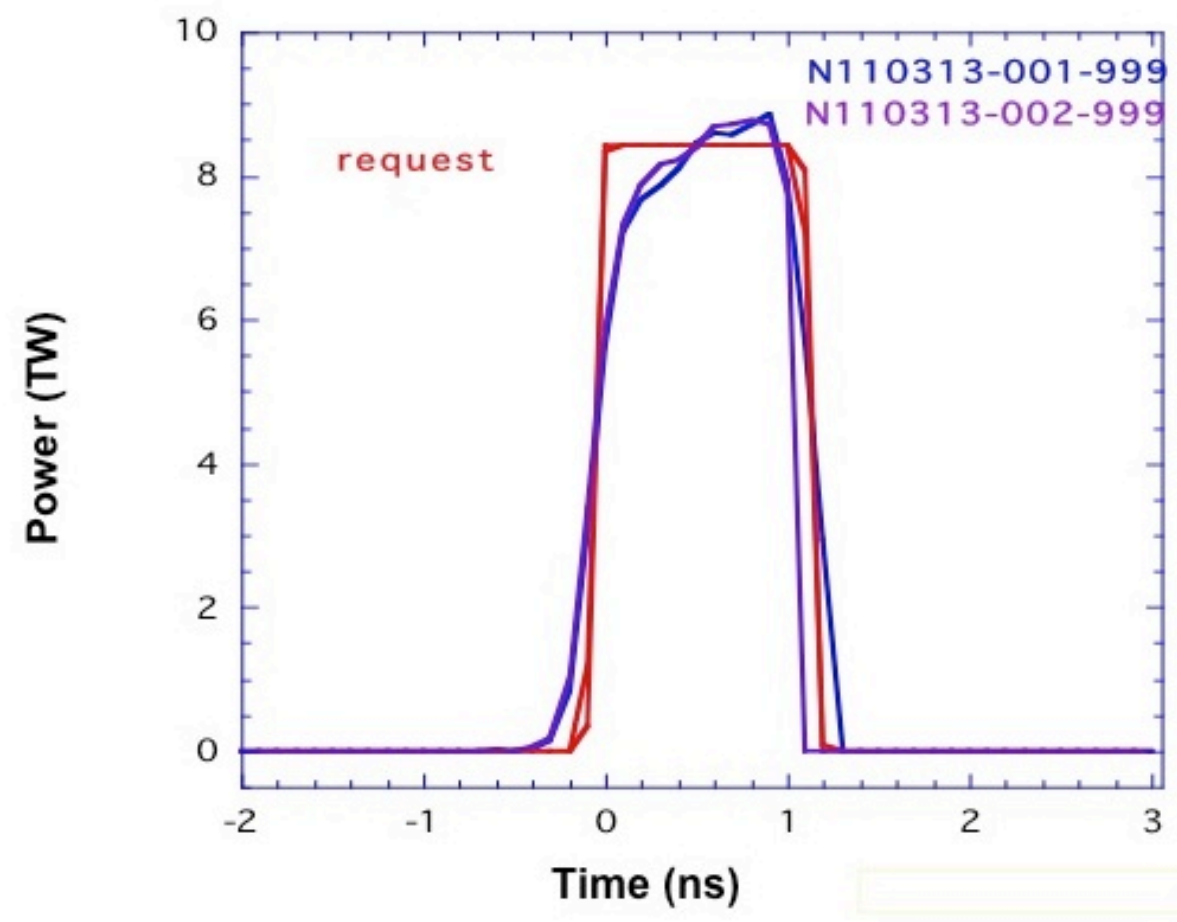

Figure 4 - requested (red) and measured (blue and purple) laser power for NIF shots N110313-001-999 and N110313-002-999.

Measurements made by various existing NIF diagnostics (DANTE-1 and -2 [14], SXIupper and lower [15], and Full-Aperture Backscatter Station (FABS) [16]) will quantify these aspects of target performance by measuring the laser energy reflected from the target via laser-plasma instabilities, as well as $x$-ray losses from the target LEH $[5,17]$. In particular, the two DANTE systems will quantify burn-through time and flux levels for $x$ rays emerging through the target wall. These measurements will be useful in the final design of our fully instrumented, full-system Data Campaign. Two halfraums were shot in the first phase of our Energetics Campaign in March 2011; the spherical targets had wall thickness of 15.9 and $12 \mu \mathrm{m}$ (the latter was out of conformance with our pointdesign value of $7 \mu \mathrm{m})$. The laser power used to drive the $12 \mu \mathrm{m}$ thick target on shot N110313-001-999 and the $15.9 \mu \mathrm{m}$ target on shot N110313-002-999 is shown in Figure 4. Also shown is the requested laser pulse shape, a 1 ns flat-top pulse, to deliver $10.5 \mathrm{~kJ}$ of energy to the target. The two measured pulses delivered 10.1 and $10.2 \mathrm{~kJ}$ of drive respectively. Thus, the drive energy for both shots was within $4 \%$ of our request, and, as can be seen in the figure, the reproducibility of the pulse shapes is excellent. 
The FABS system measures the reflected laser light [16] on NIF by using streaked spectrometers, fast and slow photodiodes, near-field cameras, and time integrated spectrometers to measure independently the characteristics of both the laser light scattered from ion-acoustic waves and from plasma-electron waves [13] in the target plasma for each of the four incident laser beams in our drive quad. The measurement is accurate to $\pm 18 \%$ over a range of backscattered energy from $5 \mathrm{~J}$ to $5 \mathrm{~kJ}$. Based on the measured laser energy reflected from our two targets, the laser-to-target coupling was 93\% of the delivered laser energy. This is excellent coupling, and provides the energy source that will drive blast phenomena in the full EPEC systems to be used in the Data Campaign.

Figure 5 shows the DANTE-1 measurement for the radiation environment inside our two EPEC halfraums. The DANTE-1 diagnostic [14] sees the radiation that escapes from
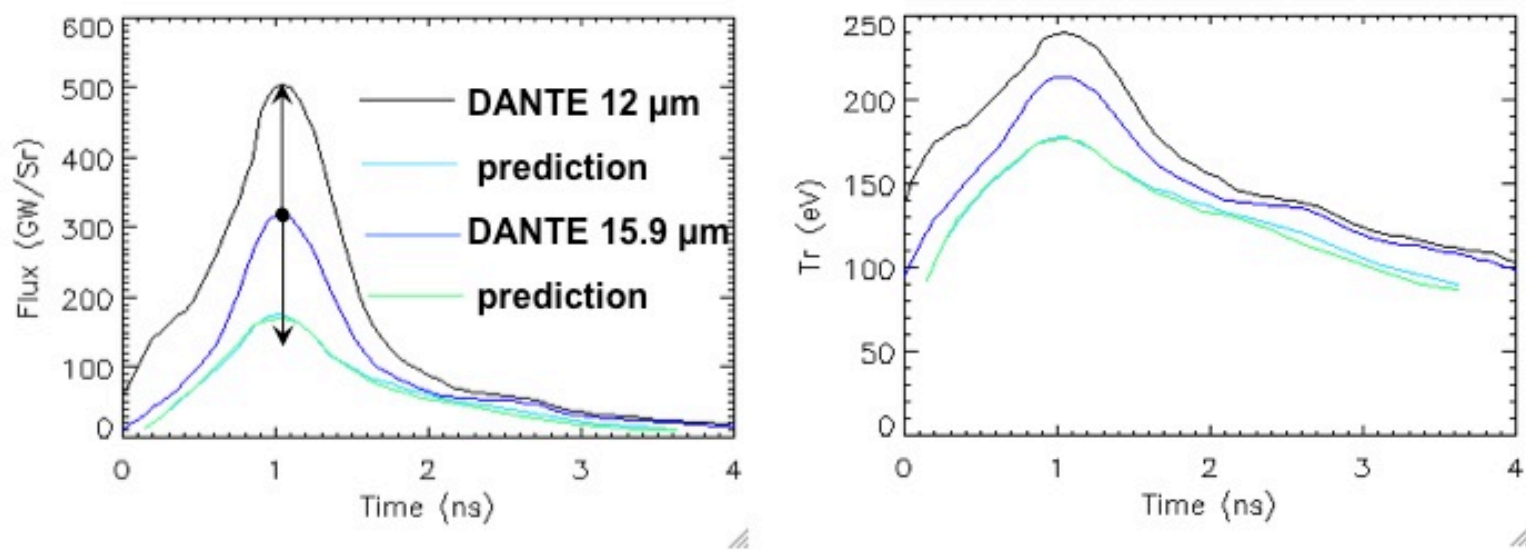

Figure 5 - DANTE-1 flux traces (left) and radiation temperature traces (right) for NIF shots N110313-001-999 (12 micron target, black) and N110313-002-999 (15.9 micron target, blue). Also shown are the predictions for these two targets (green and cyan) as given by the LASNEX radiation-hydrodynamics code.

the halfraum's LEH. Note, the peak flux in the measurement comes at $\approx 1$ ns, which is the end of the laser pulse; the flux continues to be emitted for several nanoseconds as the halfraum cools and disassembles. The right-hand panel in Fig. 5 shows the radiation temperature versus time in the EPEC halfraums; the radiation temperature is related to the flux by $T_{r}=(F / \sigma)^{1 / 4}$, where $T_{r}$ is the radiation temperature, $F$ is the measured $x$-ray flux and $\sigma$ is the Stephan-Boltzmann constant. Also shown in Fig. 5 is the predicted flux versus time for our silver halfraum and $10.5 \mathrm{~kJ}$ of laser drive delivered in a $1 \mathrm{~ns}$ square 
pulse. The prediction is done with our two-dimensional, axi-symmetric radiationhydrodynamics code LASNEX [18], and indicates that there should be no measurable difference for the internal radiation environment in the halfraums for the two different wall thicknesses. Clearly the measured data show that this is not the case, with the peak flux from the $12 \mu \mathrm{m}$ target being $3.5 \times$ that of the prediction, and the peak flux from the $15.9 \mu \mathrm{m}$ target being $2 \times$ that of the prediction. The pre-shot simulations (and post-shot analysis) have been run with what is called the High Flux Model (HFM) $[19,20]$, which accounts for state-of-the-art atomic physics and highly charged ion emission models, as well as sophisticated heat conduction models to simulate the evolution of the plasma and radiation environment inside the target. The HFM has been extensively benchmarked against energetics measurements in large-scale gold hohlraum [20]. At the moment we cannot explain why the measured fluxes (i) seem to depend on halfraum-wall thickness, and (ii) diverge so significantly from the simulations. However, one hypothesis that may explain the phenomena in Fig. 5 is that flux from the interior of the cavity is burning through the halfraum wall and contributing to the observed DANTE-1 signals. If this hypothesis is valid, it would explain both the discrepancy in the predicted peak-flux levels as well as the apparent dependence of halfraum flux on wall thickness. We are in the process of checking this hypothesis with a detailed ray-tracing analysis of the detector's line of sight to the halfraum.

Two-dimensional x-ray pinhole images of the x-ray energy emitted through the target wall during shot N110313-001-999 are shown in Fig. 6 . The images are taken with the SXI-U pinhole camera [15] through filters designed to pass only $\mathrm{x}$-rays with energies greater than $\approx 3000 \mathrm{eV}$. In addition to the blast energy from the expansion of the vaporized target material, $x$-ray energy that is emitted through the target wall will interact with the atmosphere in the full EPEC system creating a miniature fireball (see $\S$ IV.C). The x-ray flux through the target wall increases inversely with wall thickness. We plan to finish the Energetics Campaign by measuring the $x$-ray flux through the walls of two $7 \mu \mathrm{m}$ thick spherical targets. 


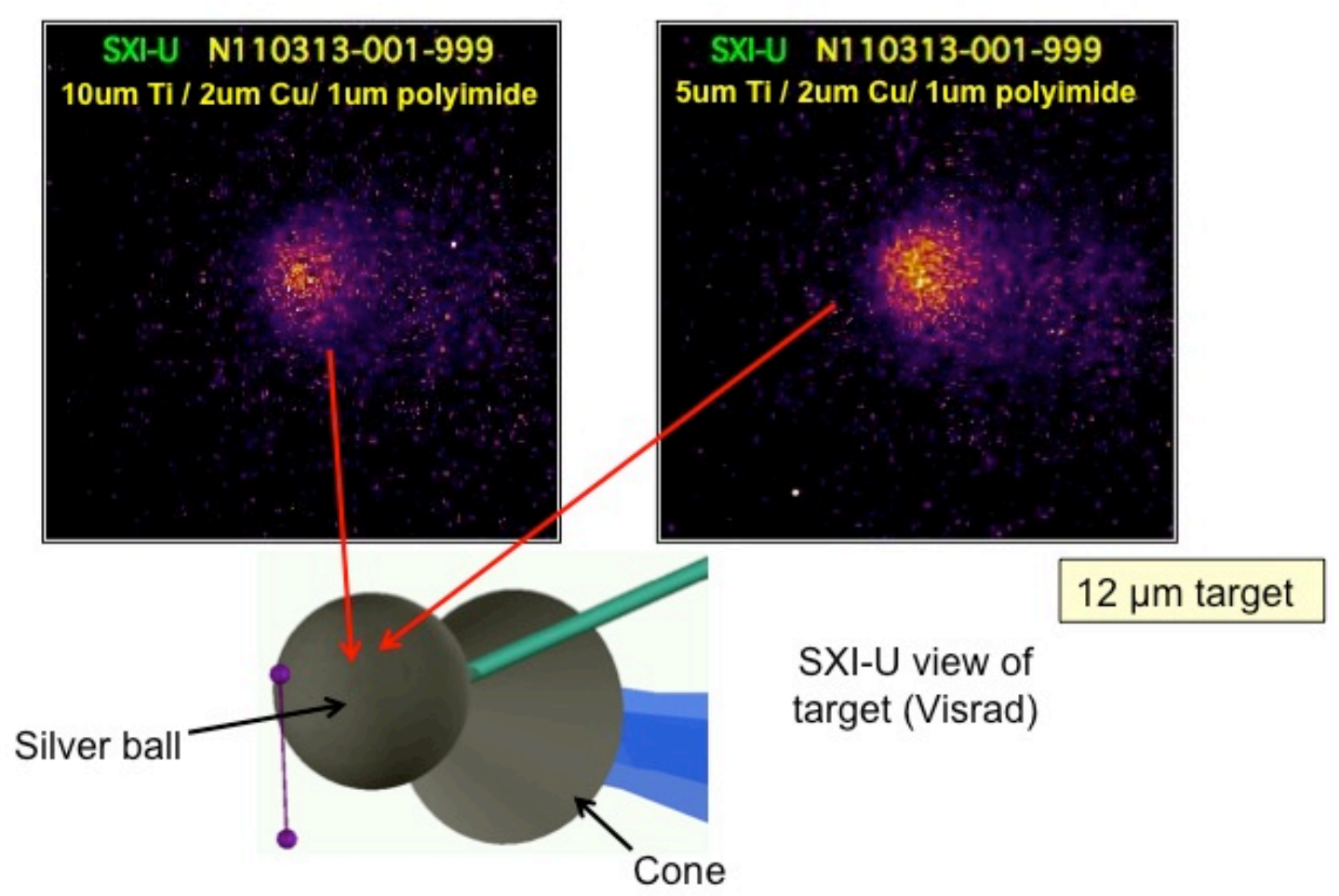

Figure 6 - SXI-U sees target package x-ray burn through. (top) Pinhole images of the EPEC halfraum target taken from a camera that views the target as shown in the schematic figure below the images. The images are from pinholes with (left) thicker and (right) thinner filter material (as listed on the image) resulting in the brighter signal in the right image. 


\section{IV.B. Data Campaign}

In our Data Campaign, we will make a proof-of-principle measurement showing that blast effects, which are driven by a laser at nuclear-weapon energy densities, can be measured in a controlled, macroscopic, pressurized environment. We will measure the ground shock and dynamic atmospheric overpressure in our EPEC test assembly. We will also measure the optical power emitted by the $x$-ray deposition from our laser target in the test atmosphere. These proof-of-principle measurements will be made for at least one scaled height of burst above a ground surrogate material.

\section{IV.B.1. EPEC Assembly}

In our Data Campaign the conical skirt of the target (see Fig. 3) is placed on the end of a stainless steel cone. The cone is the interface for the EPEC target and the NIF target-chamber vacuum, which is typically $10^{-5} \mathrm{mTorr}$. The cone is part of an airtight vessel pressurized to one atmosphere in which the EPEC experiments will take place. The pressurized EPEC system is inserted into the NIF chamber before a shot through one of the facility's diagnostic instrument manipulators (DIMs), and withdrawn afterwards. The vessel contains the one atmosphere of pressure in the vacuum of the NIF target chamber until the target is destroyed, at which point the gases vent into the NIF target chamber. The target on the tip of the cone is suspended at various heights above our ground simulant, which in this case is a 6" diameter block of BK7 (borosilicate) glass. The system is shown schematically in the $10 \mathrm{~m}$ diameter NIF target chamber in Figure 7, along with a single quad of NIF beams (blue beams from the bottom of the figure) entering the cone that positions the target above the glass surface. Modeling of the vessel is discussed in Appendix A.

The integrated EPEC diagnostic system for the Data Campaign consists of two major components: a 6.00 " square, 56" long aluminum vacuum enclosure called an air box (with walls 0.500 " thick), and a 12.00 " outer diameter, 20.5" length polycarbonate cylinder. The assembly is shown in Fig. 8. The mounting rails on the side of the air box serve as the interface between the diagnostic assembly and the DIM cart/boat assembly (shown extended into the NIF target chamber in Fig. 7). The mounting rails use tooling balls to align to a registered location in the DIM cart/boat assembly, which can be inserted by the DIM positioning system to a precise location in the NIF chamber. For the full EPEC assembly, the Opposed Port Alignment System (OPAS) will allow the diagnostic package to be positioned inside the NIF chamber to a tolerance of \pm 100 um with respect to target chamber center. The system will be aligned by using a set of fiducial monuments on the cone structure that supports the halfraum target (the beam channel in Fig. 10). The fiducial positions will be metrologized using the same optical technique used in the Energetics Campaign target metrology. 


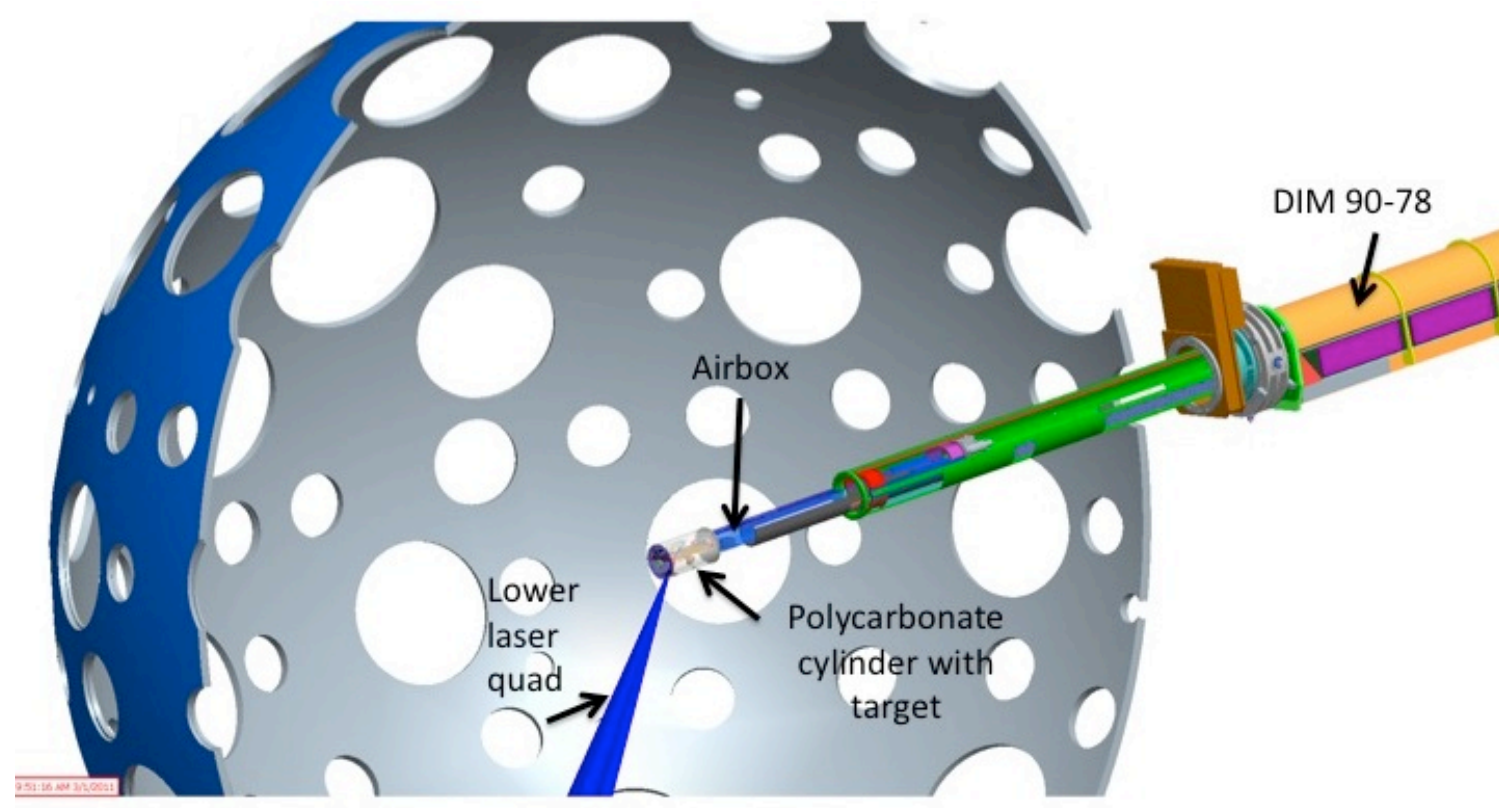

Figure 7 - EPEC pressure vessel for the Data Campaign is shown inserted into the $10 \mathrm{~m}$ diameter NIF target chamber. The pressure vessel is mounted on the front end of the air box that contains the photo-multiplier tube diagnostic as well as connections for ground-shock and air-blast sensors. 


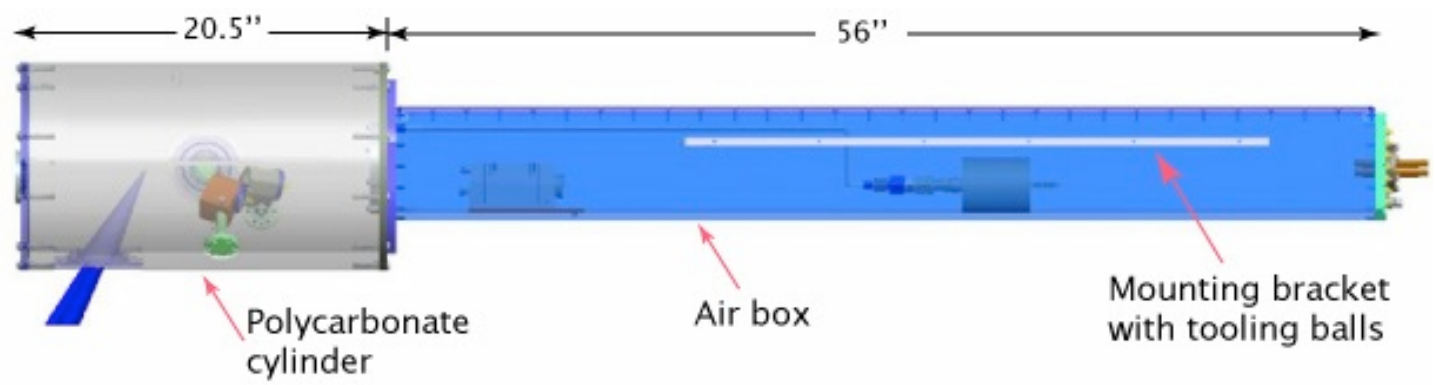

Figure 8 - The EPEC diagnostic assembly from Fig. 7 is shown here mounted on a NIF air box. The EPEC polycarbonate cylinder is the clear part on the left of the figure. A single quad of NIF laser beams is seen entering from below and to the left. Two of the EPEC air-blast sensors are visible in the cylinder. The air box is the blue rectangle that extends to the right in the figure. The housing for the EPEC PMTs is also shown in the figure.

The walls of the polycarbonate cylinder shown in Fig. 8 are 1.00" thick. During the experiment the cylinder will be filled with a gas mixture (discussed below) at $1 \mathrm{~atm}$ pressure, and will see a very significant pressure spike up to 3000 psi for approximately a microsecond. To maintain the integrity of the cylinder during a shot sequence, the cylinder has 1.00" thick aluminum end plates that are each held on with $243 / 8-16$, grade 9 , socket head cap screws. The end and side alignment viewports are made of $.500 "$ "thick, 2.00" diameter polycarbonate, and are each held in place with 16 5/16-18, grade 9 , socket head cap screws. The target cone is made of 316 stainless steel. It is conical with an 8.5 degree taper over approximately 5.00 " inches. It has a wall thickness of $0.120^{\prime \prime}$. It is also held onto the cylinder with $165 / 16-18$, grade 9, socket head cap screws. The cylinder, fully instrumented will weigh approximately 71 pounds. The full diagnostic, when mounted on the air box, will weigh slightly less the 240 pounds. 


\section{IV.B.2. EPEC Sensors}

Three ground-shock sensors will be embedded at depths of 5, 5 and $8 \mathrm{~cm}$ in the block of BK7 glass ${ }^{3}$. A photograph of the machined block of glass is shown in Figure 9; note the recesses for the three ground shock sensors at different depths and orientations in the right panel in Fig. 9. Two air-shock sensors at $\approx 10$ and $15 \mathrm{~cm}$ from
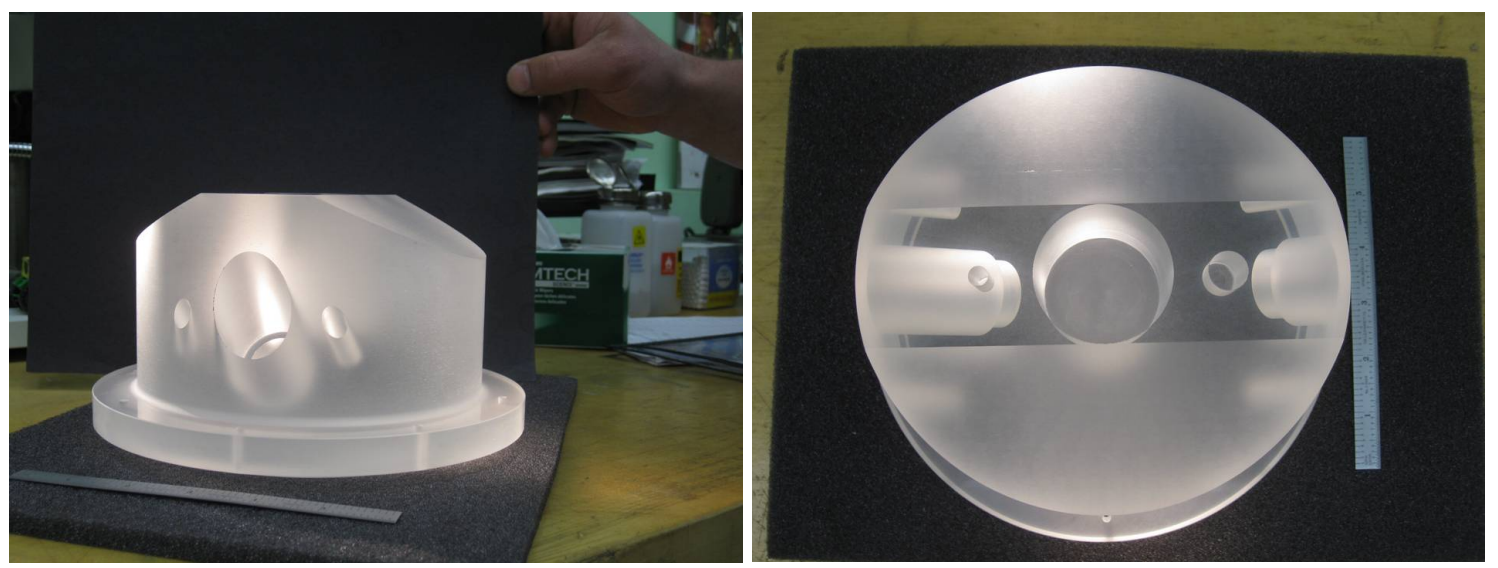

Figure 9 - The EPEC BK7 glass ground simulant (left) side view and (right) top view. One can see in the image a 6" ruler showing the top diameter. One can also see in the top view the three channels machined into the glass for the three ground shock-sensors.

the target will also be present in the EPEC assembly. Further, the pressure vessel will be mounted to the front end of an "air box" that contains two photomultiplier tubes (PMTs) that will measure the optical light curve (visible radiation versus time) produced by the interaction of the target $x$ rays with the surrounding atmosphere. X-ray flux from the laser-heated halfraum-target will not be measured in the Data Campaign; it will however have been measured during the Energetics Campaign. A closer view of the EPEC system with the glass block and the pressure sensors is shown in Figure 10. The signals from the various sensors will be routed through the DIM to NIF's diagnostic mezzanine, where they will be divided into multiple channels with different attenuations and digitizer full-scale settings to increase the dynamic range of the digitized signals. The settings of the light filters, PMT high-voltage inputs, and attenuators will be determined from data measured in our Energetics Campaign.

\footnotetext{
${ }^{3}$ The block of glass was procured from Schott Glass Manufactures, and then precision ground at Insaco Inc. in Quakertown, PA. See http://www.insaco.com/ for company details.
} 


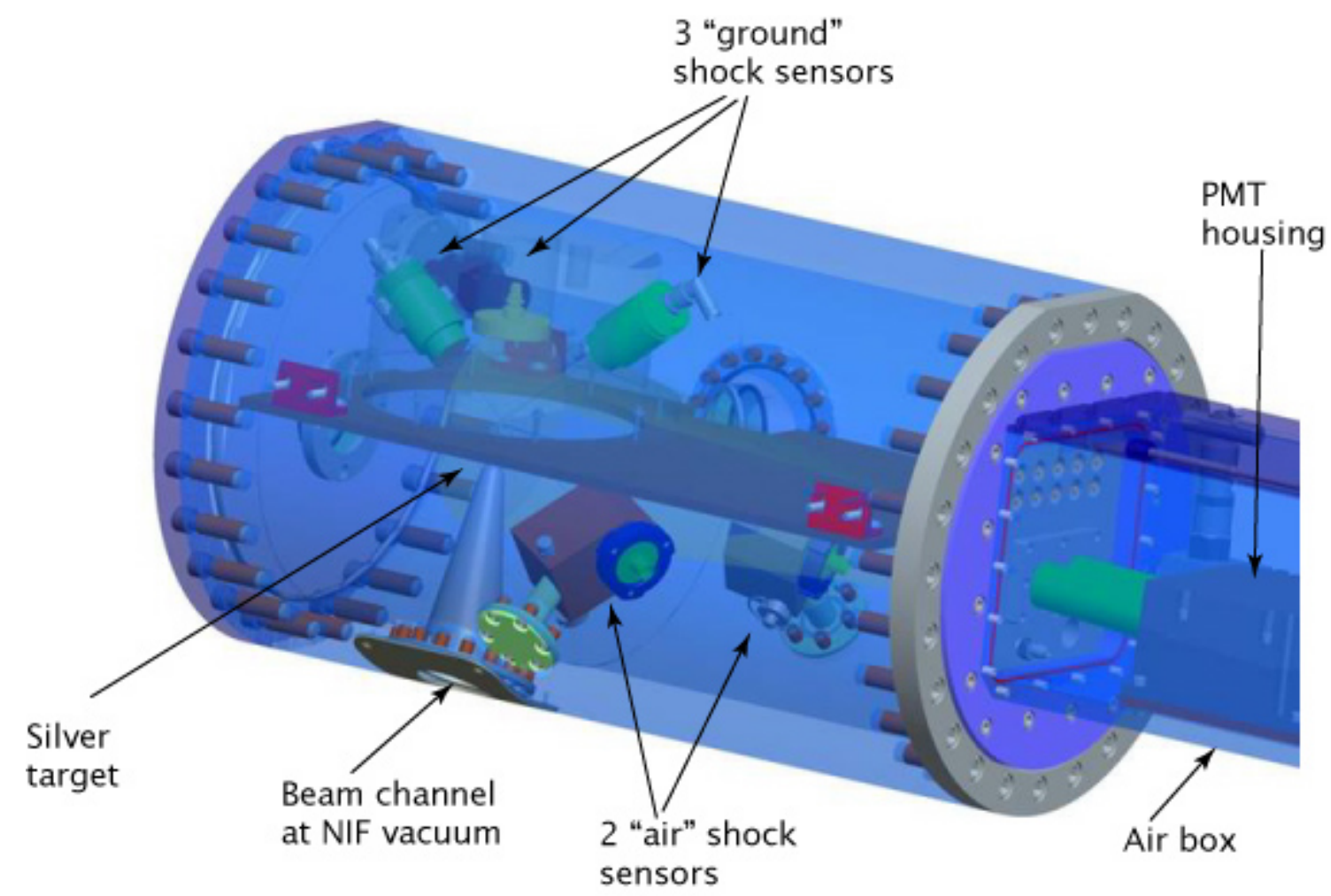

Figure 10 - CAD model of the EPEC enclosure mounted to the NIF air box that contains the PTM-based optical light diagnostic. Note the BK7 glass block from Fig. 8 has been rendered nearly invisible in the CAD model making the three ground-shock sensors visible. The NIF laser will enter the system through the dark cone at the bottom of the cylinder.

The sensors that will be used to measure the ground shock and the dynamic overpressure in the EPEC atmosphere are being provided by KTech Corporation ${ }^{4}$. They will be piezoelectric stress gauges [21] that will measure the stress rate as the waveform passes the sensor location. Two types of detectors will be used: a slower PVDF (polyvinylidene fluoride) gauge and a quartz-based faster gauge. Four PVDF gauges will be used, with two as dynamic-overpressure sensors in the EPEC atmosphere and two as ground-shock sensors in the BK7. Additionally, one quartz-based gauge will be mounted in the BK7. The sensor, either the PVDF or quartz material, is sandwiched between two electrical leads, a gold lead on top (closer to the source) and a platinum lead underneath. The overlap of the metallic leads creates the active area of the sensor, which is $\approx 5 \mathrm{~mm} \times 5 \mathrm{~mm}$, see Fig. 11. Care has been taken to set the active area of the gauges as close to normal to the propagating blast waves as possible in order to avoid

${ }^{4}$ See www.ktech.com/ for company details 
having a finite transit time across the gauge's active area, which would reduce the signal's rise time. Pre-shot sensor calibration is being done on the assembled and installed gauges on a gas gun at the KTech facility in Albuquerque, NM. The sensors' ranges of linear operation and characteristic responses will be calibrated against a known shock pressure for the actual medium (BK7 glass, air) in which the sensors will have to perform. The sensors are electrically isolated from their housings by spacer layers of an insulating plastic. The sensor itself and the insulating layers are protected from stray light and electromagnetic pulses by an aluminum-flashed Teflon film that is bonded to the sensor housing with a NIF-approved (i.e., vacuum compatible) silver epoxy (Chomerics ${ }^{\mathrm{TM}}$ silver epoxy).

(a)

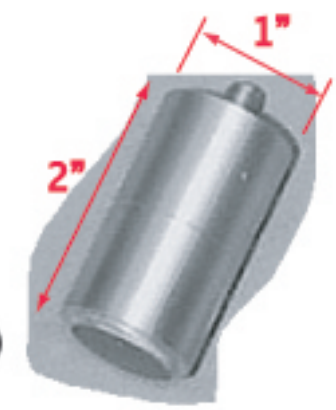

(b)

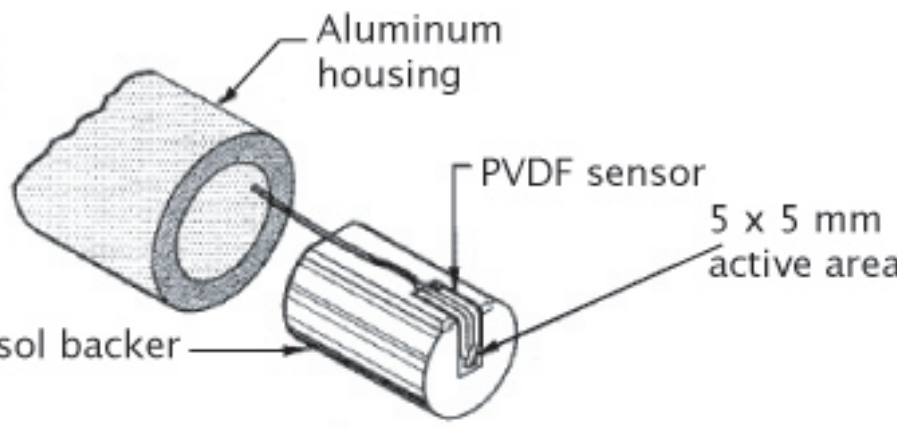

Hysol backer

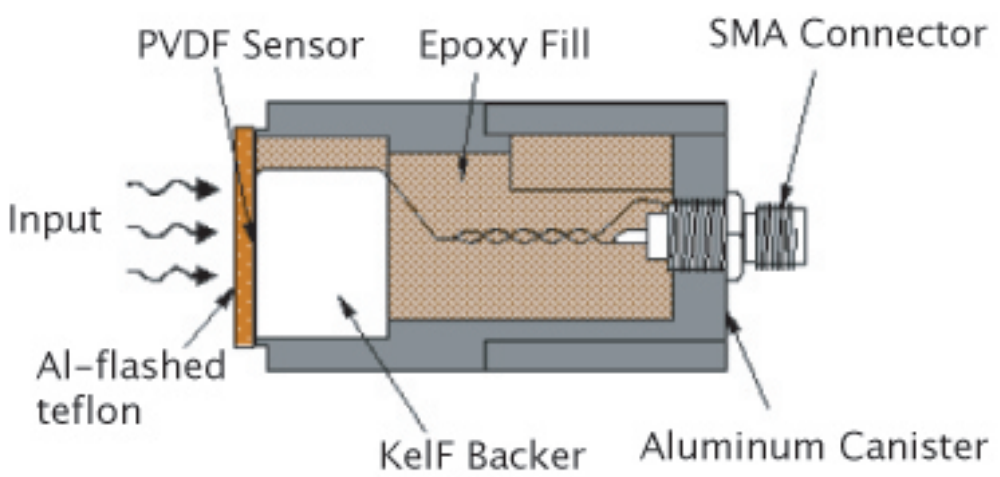

(c)

Figure 11 - (a) Photograph of Ktech PVDF shock gauge assembly showing the outer dimensions and the SMA connector at the back of the gauge. (b) Exploded view of Ktech gauge active area showing the PVDF film sensor and the crossed-electrode active area on the face of the gauge. (c) Cross section of one of the PVDF shock gauges showing the KelF backer used in the EPEC experiments, and the Hysol epoxy fill in the aluminum gauge housing. 


\section{IV.C - Scaled Fireball Properties}

In addition to the energy that drives the blast and shock phenomena described above, we expect a significant portion of the laser energy that couples to our halfraum target will emerge from the EPEC system as radiation in the visible waveband. Following Glasstone and Dolan [1] (\$1.22-1.27 and 1.77), broadly speaking, the energy from a nuclear weapon can be assigned to one of three categories: kinetic energy, i.e., energy of motion of electrons atoms, and molecules as a whole; internal energy of these particles; and thermal radiation energy. In a generic fashion, a nuclear weapon emits a major fraction, $65-80 \%$ of its yield as radiation, which includes electromagnetic radiation across all energies from infrared to the $x$ ray. This radiation interacts with the atmosphere surrounding the hot weapon debris and creates a region of ionized plasma or a "fireball". As the weapon debris expands, eventually the debris shockwave outruns the fireball creating an ionized layer of air at the shock front. Stripped electrons in this layer obscure the visible radiation from the hot region within. As the shock expands radially outward, it becomes increasingly transparent to optical radiation. Hence, there

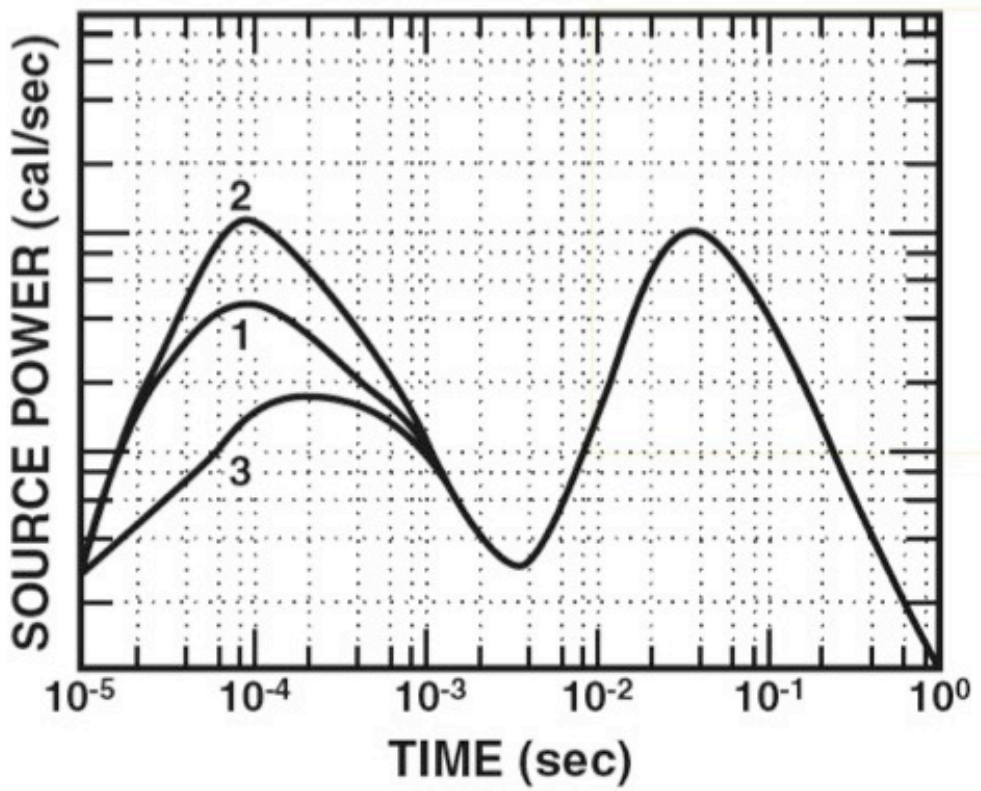

Figure 12 - Illustrative Fireball Thermal Power Versus Time for a 1-KT, Low Altitude Nuclear Explosion, Showing Variability of the First Pulse. Taken from Ref. 22.

is a characteristic dynamic quality to the optical emission from a nuclear weapon's fireball, as shown in Fig. 12, which is taken from Ref. [22].

Since we are creating an environment in our EPEC system that shares both a nuclear weapon's energy density and specific energy, we expect that the $x$ rays that escape from 
our target into our test-system atmosphere will create a miniature fireball. We expect there will be interplay of radiation from the hot plasma and absorption from the atmospheric shock in our EPEC system. As a result, we have designed a diagnostic system to measure the dynamics of optical radiation from our mini-fireball. We do not expect the dynamics of the fireball power to span the range shown in Fig. 12, but based on radiation hydrodynamic simulations [18], we do expect to see some indication of the interplay between $\mathrm{x}$-ray deposition and shock-induced opacity giving rise to the characteristic shape shown in Fig. 12.

We have scaled the spatial and temporal dimensions in our experiment to preserve the hydrodynamic evolution of shock pressures and shock velocities in our surrogate "ground" and "air" media. We have kept thermodynamic and equation-of-state properties as similar as possible in our scaled system to the same quantities in the reference system. Thus, the atmospheric pressure in our system remains initially at $1 \mathrm{~atm}$. One parameter that is not scaled by the cube-root scaling of the ratio of our drive energy to the reference-system energy (i.e., the weapon yield) is the absorption length of the $x$-ray emission from our laser-heated target in our surrogate atmosphere. We have affected this scaling by increasing the opacity in the spectral band of the halfraum emission of our surrogate atmosphere (at a pressure of $1 \mathrm{~atm}$ ) by adding heavy impurities, namely $\mathrm{Ar}, \mathrm{Kr}$ and $\mathrm{Xe}$ to the basic $\mathrm{N}_{2}$ and $\mathrm{O}_{2}$. The gases in the EPEC system are a blend of $44 \% \mathrm{~N}_{2}, 21 \% \mathrm{O}_{2}, 20 \% \mathrm{Ar}, 10 \% \mathrm{Kr}$ and $5 \% \mathrm{Xe}$, which is necessary to increase the atmosphere's opacity to $x$-rays consistent with the hydrodynamically scaled spatial dimensions in the EPEC system. This surrogate atmosphere has been designed with LLNL's radiation-transport code LASNEX [18] and library of opacity data. Thus, to within the limits that our EPEC atmosphere exhibits the behavior of a so-called polytropic gas [3], we have preserved the hydrodynamic similarity of the atmospheric response to the halfraum explosion while at the same time creating the conditions for scaled $x$-ray deposition that will give rise to a $x$-ray heated fireball in our EPEC atmosphere.

The diagnostic that will measure the optical light curve of from our halfraumproduced fireball consists of two Hamamatsu model R5946 Photo-Multiplier Tubes (PMTs) behind various filters in our NIF air box. Before any full-system shots in our Data Campaign, we will qualify the PMT diagnostic against contamination from stray light and the effects of electromagnetic interference, and integrate it into the NIF control system. The PMTs will be housed in a vacuum-tight air box (Figure 13) attached to a DIM. The DIM-insertable air box will have filters to block laser light and filters to attenuate the optical light of interest to avoid saturation. Further control of the signal from the PMTs will be accomplished by the high-voltage input to the PMTs. The signals from the PMTs will be routed through the DIM to the diagnostic mezzanine, where they will be divided 
into multiple channels with different attenuations and digitizer full-scale settings to increase the dynamic range of the digitized signals.

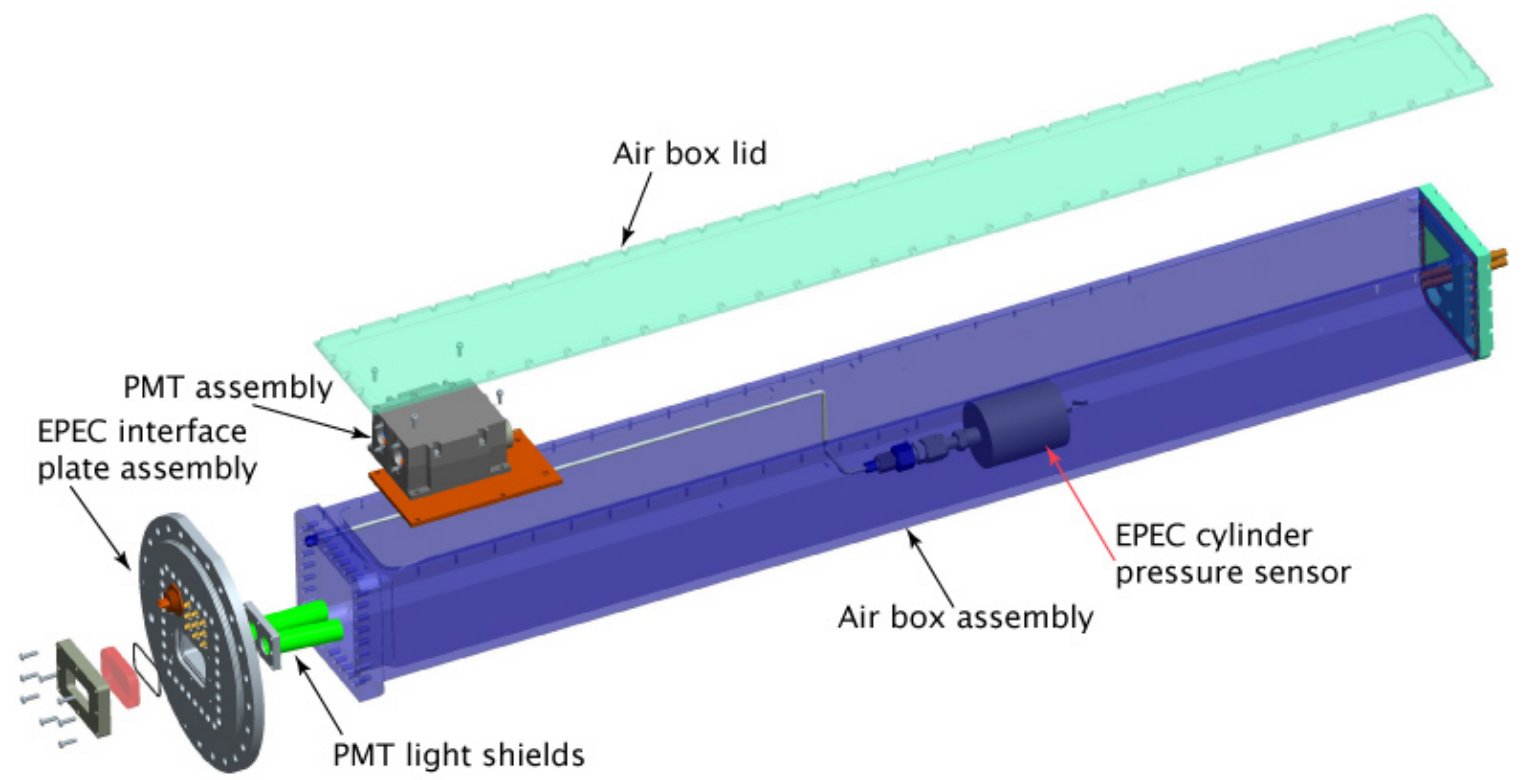

Figure 13 - An exploded view of the EPEC photo-multiplier tube diagnostic assembly components and a custom-designed NIF air box with an interface plate unique to the EPEC experiment. The interface plate is designed to withstand greater loads than are typically experienced on NIF diagnostics.

\section{Summary}

We have designed an experimental system to be fielded at the NIF laser in 2011. The system will create a high-energy-density environment that will have the same energy density and specific energy as a low-yield nuclear weapon. The system will allow us to study in a controlled manner the partitioning of the system energy between ground shock and dynamic overpressure for known heights of burst. We will have multiple channels in both the ground simulant and in our test atmosphere to reconstruct the stress waveforms at multiple distances from the blast point. Additionally, we will measure the dynamics of optical radiation that results from reprocessing of target $x$ rays in our test atmosphere. The ultimate deliverable from this system will be a highly accurate suite of data that can be used for validating

We would like to acknowledge contributions to this project from Doug Vogt, Otis Walton, Brian Bonner, Victor Karpenko, Dana Hargrove and Ted Perry at LLNL, Emilio Giraldez at General Atomics and Tom Kiess at NNSA/NA-22. This work performed under the auspices of the U.S. Department of Energy by Lawrence Livermore National Laboratory under Contract DE-AC52-07NA27344. 


\section{References:}

[1] "The Effects of Nuclear Weapons (Third Edition)", compiled by S. Glasstone and P. Dolan, 1977, US Department of Defense and US Department of Energy.

[2] L. D. Landau, E. M. Lifshitz, Fluid Mechanics $2^{\text {nd }}$ Edition, Course of Theoretical Physics Volume 6 (Butterworth-Heinemann, Oxford UK, 1987).

[3] D. Ryutov, R. P. Drake, J. Kane, E. Liang, B. A. Remington, W. M. Wood-Vasey, "Similarity Criteria for the Laboratory Simulation of Supernova Hydrodynamics", The Astrophysical Journal 518, 821 (1999).

[4] R. I. Klein, C. F. Mckee, P. Colella, "On The Hydrodynamic Interaction Of ShockWaves With Interstellar Clouds .1. Nonradiative Shocks In Small Clouds" The Astrophysical Journal 420, 213 (1994); R. I. Klein, K. S. Budil, T. S. Perry, D. R. Bach, "The Interaction Of Supernova Remnants With Interstellar Clouds: Experiments On The Nova Laser", The Astrophysical Journal 583,245 (2003).

[5] N. B. Meezan, et al., "National Ignition Campaign Hohlraum Energetics", Phys. Plasmas 17, 056304 (2010).

[6] I. Lomov, "Simulation of dense and dilute multiphase compressible flows with Eulerian-Lagrangian approach", LLNL technical report UCRL-PROC-231305, in Proc. of 6th International Conference on Multiphase Flow, ICMF 2007, Leipzig, Germany, July 9-13, 2007.

[7] Y. Kanarska, I. Lomov, L. Glenn, T. Antoun, "Numerical simulation of cloud rise phenomena associated with nuclear bursts", Annals of Nuclear Energy 36, 14751483 (2009);

[8] I. Lomov, R. Pember, J Greenough, B. Liu, "Patch-based Adapative Mesh Refinement for Multimaterial Hydrodynamics", LLNL technical report UCRL-CONF2164444, in Proc. of Joint Russian-American Five-Laboratory Conference on Computational Mathematics, Vienna, June 19-23, 2005.

[9] Lawrence Livermore National Laboratory, NIF National Ignition Facility \& Photon Science Bringing Star Power to Earth. [Online, cited: September 27, 2010.] https://lasers.Ilnl.gov/.

[10] C. A. Haynam, et al., "National Ignition Facility laser performance status", Appl. Optics 46, 3276-3303 (2007).

[11] E. M. Giraldez, M. T. Vu, P. Mirkarimi, K. B. Fournier, M. E. Schoff, H. Huang, J. S. Jaquez, and E. C. Losbanos, "Fabrication of Thin Wall Silver Spherical Targets for EPEC Experiments on NIF", presentation to the $20^{\text {th }}$ Target Fabrication Meeting, Honolulu, HI, Oct. 2-7, 2011.

[12] P. B. Mirkarimi, N. E. Teslich, J. D. Sain, M. J. Wilson, M. J. May, J. A. Emig, R. J. Wallace, K. B. Fournier, E. M. Giraldez, M. E. Schoff, and H. Huang, "The Metrology Challenge in Characterizing Thin, Hollow, Silver Spherical Targets for EPEC Experiments on NIF", presentation to the $20^{\text {th }}$ Target Fabrication Meeting, Honolulu, HI, Oct. 2-7, 2011. 
[13] W. L. Kruer, The Physics of Laser Plasma Interactions (Westview, Boulder, CO, 2003).

[14] E. L. Dewald, K. M. Campbell, R. E. Turner, J. P. Holder, O. L. Landen, S. H. Glenzer, R. L. Kauffman, L. J. Suter, M. Landon, M. Rhodes, and D. Lee, "Dante soft x-ray power diagnostic for National Ignition Facility", Rev. Sci. Instrum. 75, 3759 (2004).

[15] M. Landon, J. Koch, S. Alvarez, P. Bell, F. Lee, and J. Moody, "Design of the National Ignition Facility static x-ray imager", Rev. Sci. Instrum. 72, 698 (2001).

[16] D. H. Froula, et al., "Full-aperture backscatter measurements on the National Ignition Facility", Rev. Sci. Instrum. 75, 4168 (2004). J. Moody, et al., "Optical backscatter measurements to study laser plasma interactions on NIF", Bull. Am. Phys. Soc. 54, 142 (2009).

[17] K. B. Fournier et al., "Multi-keV x-ray source development experiments on the National Ignition Facility", Phys. Plasmas 17, 082701 (2010).

[18] G. Zimmerman and W. Kruer, "Numerical Simulation of Laser-Initiated Fusion", Comments Plasma Phys. Controlled Fusion 2, 51 (1975); J. A. Harte, W. E. Alley, D. S. Bailey, J. L. Eddleman, G. B. Zimmerman, "LASNEX-A 2-D Physics Code For Modeling ICF", LLNL technical report UCRL-LR-105821-96-4 (1996).

[19] M.D. Rosen et al., "The role of a detailed configuration accounting (DCA) atomic physics package in explaining the energy balance in ignition-scale hohlraums", High Energy Density Physics 7180 (2011).

[20] R. P. J. Town et al., "Analysis of the National Ignition Facility ignition hohlraum energetics experiments" Phys. Plasmas 18, 056302 (2011).

[21] L. M. Lee, D. E. Johnson, F. Bauer, R. P. Reed and J. I. Greenwoll, "Piezoelectric Polymer PVDF Application Under Soft X-Ray Induced Shock Loading", in Shock Compression of Condensed Matter 1991, S.C. Schmidt, R.D. Dick, J.W. Forbes, D.G. Tasker (editors), 1992 Elsevier Science Publishers B.V.

[22] G. E. Barash, "Light Flash Produced by an Atmospheric Nuclear Explosion", Los Alamos Technical Report, LASL-79-84 (November 1979).

[23] I. Lomov and T. Antoun, "Simulations of Geomaterials Using Continuum Damage Models on an Eulerian Grid", LLNL technical report UCRL-CONF-206641, in Proc. of $11^{\text {th }}$ International Conference on Fracture, Turin, Italy, Mar 20 - 25, 2005.

[24] T. H. Antoun and I. Lomov, "Simulations of a Spherical Wave Experiment in Marble Using a Multidirectional Damage Model", LLNL technical report UCRL-JC154428, in Proc. of $13^{\text {th }}$ American Physical Society SCCM Conference, Portland, Oregon, July 21 - 25, 2003; I. Lomov, O. Vorobiev, T. H. Antoun, "Simulation of Shock Wave Propagation and Damage in Geologic Materials", LLNL technical report UCRL-CONF-206632, in Proc. of International Symposium on Plasticity 2005, Kauai, HI, Jan. 3-8, 2005. 


\section{A. APPENDIX}

The following is taken from Lomov [6] and Lomov, Pember, Greenough and Liu [8]. Application of GEODYN to a purely vapor-phase problem is discussed in Kanarska, Lomov, Glenn and Antoun [7]. An excellent discussion of the scale-invariant physical phenomena in Euler-scaled systems is given in Ryutov et al. [3], and the interested reader is strongly pointed there.

The hydrodynamic code used in this work, called GEODYN [6], was developed at LLNL and incorporates physical models to fully describe a broad range of phenomena including shock and thermodynamic behavior. It is an Eulerian code with adaptive mesh refinement (AMR) [8]. There are two basic types of dynamic codes, Lagrangian and Eulerian. A Lagrangian code's mesh, or background, moves with the material, so no mass flows between cells. In an Eulerian code, the mesh or background is stationary and the material is allowed to move though stationary cells. An Eulerian code is analogous to looking at a dust storm through a window whereas a Lagrangian code is analogous to floating along with an individual dust particle in the storm. The adaptive mesh means that the code has the ability to vary the level of detail of the background.

\section{A.1. Thermodynamics}

The two primary areas of physics in the GEODYN code are thermodynamics and continuum mechanics. GEODYN simplifies the matter without deviation from real experimental results by implementing the various physics constraints into a formulation based on the 1st and 2nd laws of thermodynamics. Specifically, GEODYN obeys the laws of conservation of energy, and increase in entropy or the direction of spontaneous energy in such a way that the entropy of the system always increases. Thus, the constitutive equations that GEODYN solves are required to satisfy that heat flow from hot to cold regions and that the material dissipation is nonnegative. The equations of state (EOS) used for materials in the GEODYN code typically come from LLNL's tabular LEOS library and go beyond assumptions of simple ideal gases. (For example, in our mixed EPEC atmosphere, the adiabatic exponent $\gamma=1.47$, as opposed to 1.4 that is appropriate for diatomic rigid rotors.) For solids materials, a Mie-Gruneisen equation of state can be used when a specific table is not available.

For solids, GEODYN calculates directional tensile failure in the continuum model in such a way that the code can simulate the weakening and void formation that results. [23] The model is developed within the context of a properly invariant nonlinear thermomechanical theory. A second-order damage tensor is introduced that allows simulation of weakening to tension applied in one direction, without weakening to subsequent tension applied in perpendicular directions. Porosity is used as an isotropic measure of volumetric void strain and its evolution is influenced by tensile failure. The rate of dissipation due to directional tensile failure takes a particularly simple form, 
which can be analyzed easily. Specifically, the model can be combined with general constitutive equations for porous compaction and dilation, as well as viscoplasticity.

\section{A.2. Continuum Mechanics}

The GEODYN method embraces fluids as well as elastic-plastic solids in a single Eulerian framework. The code takes advantage of excellent high-order Godunov methods for producing highly accurate and efficient solutions to shock capturing problems. GEODYN builds it numerical scheme based on a system of conservation law type equations written in the flux form in the Eulerian frame of reference:

$$
\frac{\partial U_{i}}{\partial t}+\nabla \cdot G_{i}\left(U_{1}, \ldots, U_{\ell}\right)=H_{i}\left(U_{1}, \ldots, U_{\ell}\right)
$$

The vector $U_{i}, i=1, \ldots, I$ contains all the variables that define the state of material, the flux function $G_{i}$ consists of terms which can be represented in divergent form and $H_{i}$ is the source term. The first part of the system (A.1) is common for both fluid and solid dynamics and consists of the laws of conservation of mass, momentum and energy:

$$
\begin{aligned}
& \frac{\partial \rho}{\partial t}+\nabla \cdot(\rho \vec{v})=0 \\
& \frac{\partial}{\partial t}(\rho \vec{v})+\nabla \cdot(\rho \vec{v} \otimes \vec{v}-\vec{T})=0 \\
& \frac{\partial}{\partial t}\left(\rho\left(\varepsilon+\frac{\vec{v} \cdot \vec{v}}{2}\right)\right)+\nabla \cdot\left(\rho \vec{v}\left(\varepsilon+\frac{\vec{v} \cdot \vec{v}}{2}\right)-\vec{T} \cdot \vec{v}\right)=0
\end{aligned}
$$

where $\rho$ is the density, $\vec{v}$ is the velocity vector, $\vec{v}=\left(\mathrm{v}_{\mathrm{x}}, \mathrm{v}_{\mathrm{y}}, \mathrm{v}_{\mathrm{z}}\right)$ and $\left(\varepsilon+\frac{\vec{v} \cdot \vec{v}}{2}\right)$ is the total energy, $\varepsilon$ is the internal energy per unit mass, and $\vec{T}$ is the general Cauchy stress tensor. The Cauchy stress tensor can be written in the hyperelastic form as

$$
\vec{T}=-p \vec{I}+\vec{T}^{\prime}
$$

where $p$ is the thermal pressure, $\vec{I}$ is the identity tensor, and $\vec{T}^{\prime}$ is the deviatoric stress tensor of the solid matrix

$$
\overleftrightarrow{T}^{\prime}=(1-\phi) \overleftrightarrow{T}_{S}^{\prime}
$$

where $\phi$ is the current value of porosity in the solid matrix. [22] The tensor $\vec{T}$ reduces to the thermal pressure for a gas. Equations A.2 - A.4 represent expressions of conservation of mass (the so-called continuity equation), momentum and energy, respectively, for the system being simulated and are known as the Euler equations. GEODYN has been benchmarked against measurements of shock-wave propagation in geological materials [24], and observations of cloud evolution from atmospheric nuclear tests [7]. 


\section{A.3. Cube-root scaling}

Limiting ourselves to the case of a compressible hydrodynamic fluid (i.e., without the terms in the Cauchy stress tensor specific to solid bodies), the Euler equations assume the familiar form:

$$
\begin{aligned}
& \left.\frac{\partial}{\partial t}(\rho \vec{v})+\nabla \cdot(\rho \vec{v} \otimes \vec{v})+\nabla P\right)=0 \\
& \frac{\partial}{\partial t}(E)+\nabla \cdot(\vec{v}(E+P))=0
\end{aligned}
$$

where equation (A.2) has not been changed, $P$ is the pressure from equation (A.5), and $E=\rho \varepsilon+\frac{1}{2} \rho(\vec{v} \cdot \vec{v})$ is the total energy per unit volume, with $\varepsilon$ being the internal energy per unit mass for the fluid.

Equations (A.2) to (A.4) (or equivalently (A.2), (A.7) and (A.8)) remain invariant under the transformation (bearing the subscript 1$)$ :

$$
\begin{array}{ll}
\vec{r}=a \vec{r}_{1} & t=b t_{1} \quad \rho=c \rho_{1} \\
P=\left(\frac{a}{b}\right)^{2} P_{1} & \varepsilon=\left(\frac{1}{c}\right)\left(\frac{a}{b}\right)^{2} \varepsilon_{1}
\end{array}
$$

where $a, b$, and $c$ are arbitrary positive numbers. There is a direct correspondence between any two systems satisfying equation (A.9). This is the basis of the statement from Glasstone and Dolan quoted in Section I in the discussion of equation (3). Recall that for the nuclear weapons effects case, we are looking at the pressure at a fixed distance from a blast of a given yield, and relating that to the same pressure at a scaled distance from a blast of a different yield. Noting that the pressure in a system is defined as the energy per unit volume in the system, we can relate the yield of the blast to the pressure at a point by:

$$
W=\int d V\left(\rho \varepsilon+\frac{1}{2} \rho(\vec{v} \cdot \vec{v})\right)=\int d V P=\int\left(a^{3}\right) d V_{1}\left[c\left(\frac{a}{b}\right)^{2} P_{1}\right]=\frac{a^{5}}{b^{2}} c W_{1}
$$

where $\mathrm{W}$ represents the total energy in the system. For an above-ground detonation case, we can assume a constant density in the system at the two locations, i.e., $c=1$ in equation (A.9). Thus, for two systems with different total energies, when spatial and temporal dimensions scale by the same factor (i.e., $a=b$ ), we find the scale factor $a$ is

$$
a=\left(W / W_{1}\right)^{\frac{1}{3}} \text {. }
$$

\section{A.4. Cylinder modeling}

The response of the atmosphere and the dynamic overpressures on the surfaces of the cylinder, cone, air blast sensors and BK7 glass substrate are modeled using the HYDRA (RRRHYDRA) code (Fig. XXXcylmodel). The strains in the cone, end walls and cylinder are modeled using Autodesk (RRRAutodesk) and spot-checked using DYNA3D (RRRDYNA3D). The gas mixture in the 3D HYDRA models is N2: 44\%, O2: $21 \%$, Ar: 
$20 \%$, $\mathrm{Kr}: 10 \%$, Xe: $5 \%$. This mixture is designed to give a scaled man free path for absorption of radiation. Air is about $99 \%$ diatomic; our gas mixture is $65 \%$ diatomic. From ideal gas thermodynamics, the adiabatic exponent is $\gamma=1+2 \mathrm{~N} / \mathrm{F}$, where $\mathrm{N}$ is the total number of particles, and $F$ is the total number of degrees of freedom, $F=3 \mathrm{~N}+2$ $\mathrm{N}_{\text {diatomic. }}$ Thus

$$
\gamma=1+2 /\left(3+2 * N_{\text {diatomic }} / N\right)=1+2 /(3+2 * 0.65)=1.47
$$

To mock up the pressure in the EPEC Data cylinder produced by the blast wave, using 3D HYDRA we model a $12 \mathrm{~cm}$ radius, $50 \mathrm{~cm}$ long cylinder with a $10.5 \mathrm{~kJ}$ thermal 'point' source $1.0 \mathrm{~cm}$ above the 'shelf' ground plane. Boundaries, disk, and cylinder wall are reflecting. The shelf, sensors, and long beam entry cone are modeled as very heavy ideal gas, which effectively acts as an immovable, reflecting solid. We monitor the pressure on the end plate, cone, and cone flange. The model is first checked against the Sedov-Taylor blast wave result for the case of very low background pressure and no cone. The results for the full model are consistent with the expected dynamic overpressure, which includes doubling of thermal pressure on reflection, plus a sharp impulse due to the strong ram pressure. An initially spherical blast wave is distorted by the cone, sensors, shelf, and reflections from walls (Fig. XXXcylblast). Pressure enhancement occurs where shocks converge, e.g. where the end plates meets the wall.

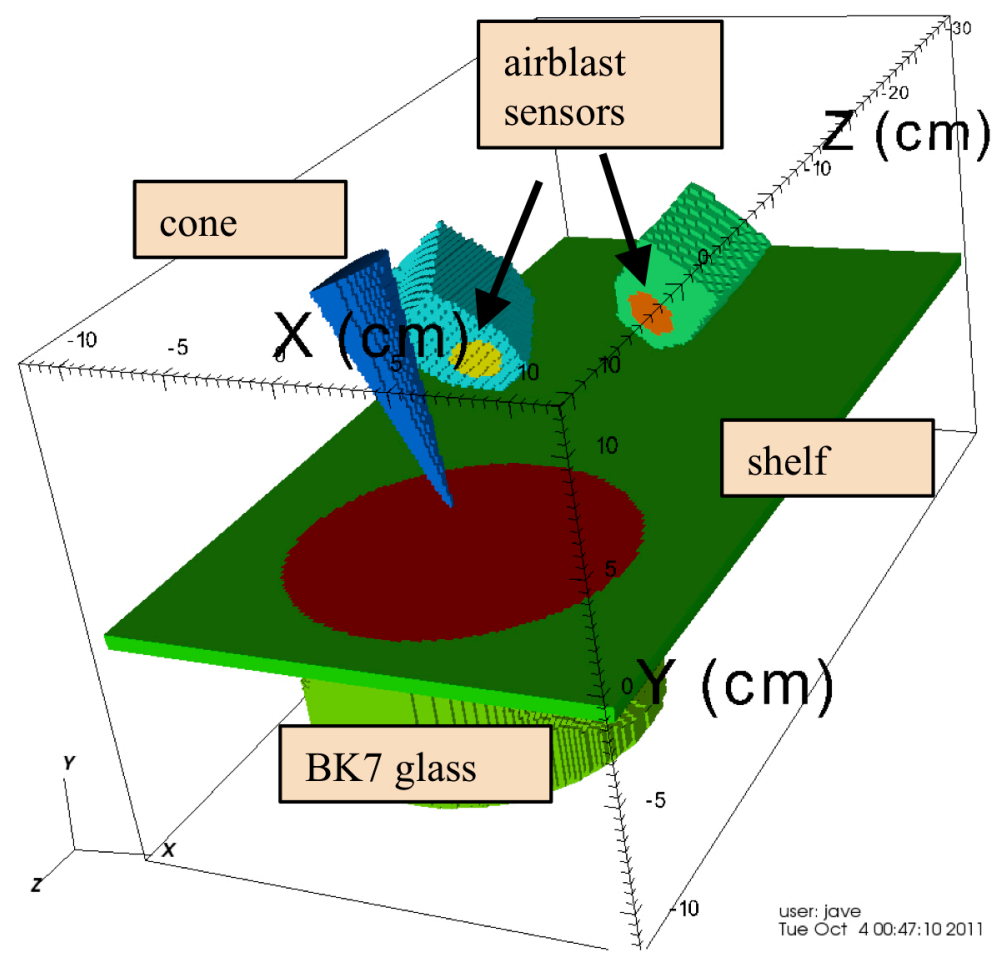


Figure XXXcylmodel. Cylinder modeling. Air, cylinder wall and end plate boundaries are not shown.

For the purpose of calibrating the airblast sensors and ground shock pressure sensors, dynamic overpressure profiles are extracted in the atmosphere next to the airblast sensors and the BK7 surface (Fig. XXXairsensor). To assess strain safety factors, dynamic overpressure profiles are extracted in the atmosphere next to the cone, the cone flange connecting the cone to the cylinder, and the aluminum end plates (Fig. XXXsf). Using these extracted profiles, Autodesk computes strain safety factors for the cylinder, cone, cone flange, and end plates, with spot checks from DYNA3D (Fig. XXXauto).

This combination of HYDRA, Autodesk and DYNA3D modeling has led to a number of refinements to the initial design of the EPEC Data cylinder, including using more bolts to connect the end plates and the cone flange to the cylinder and increasing the thickness of the long EPEC Data beam entry cone connecting the outside chamber vacuum to the source halfraum target.
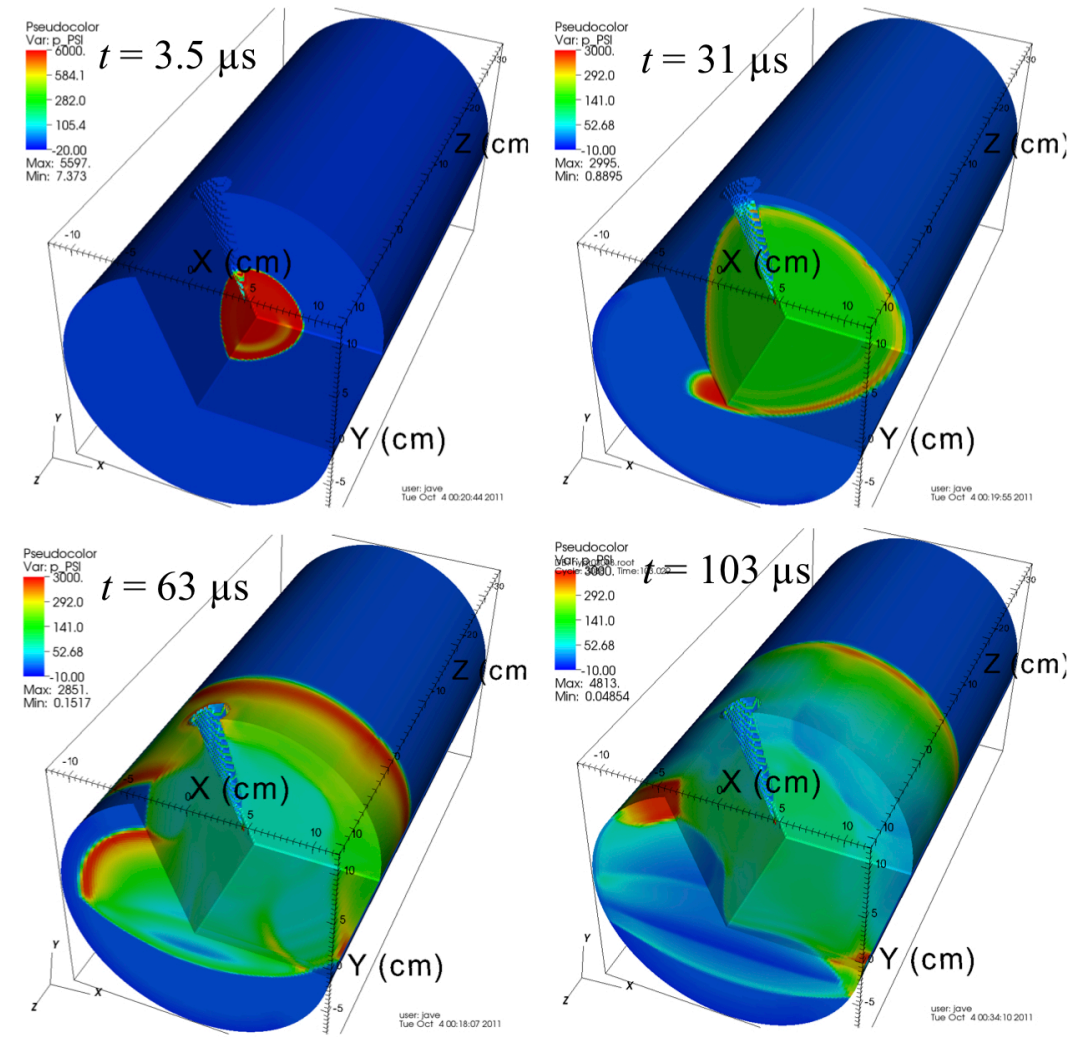

Figure XXXcylblast. Blast wave interactions inside cylinder. 

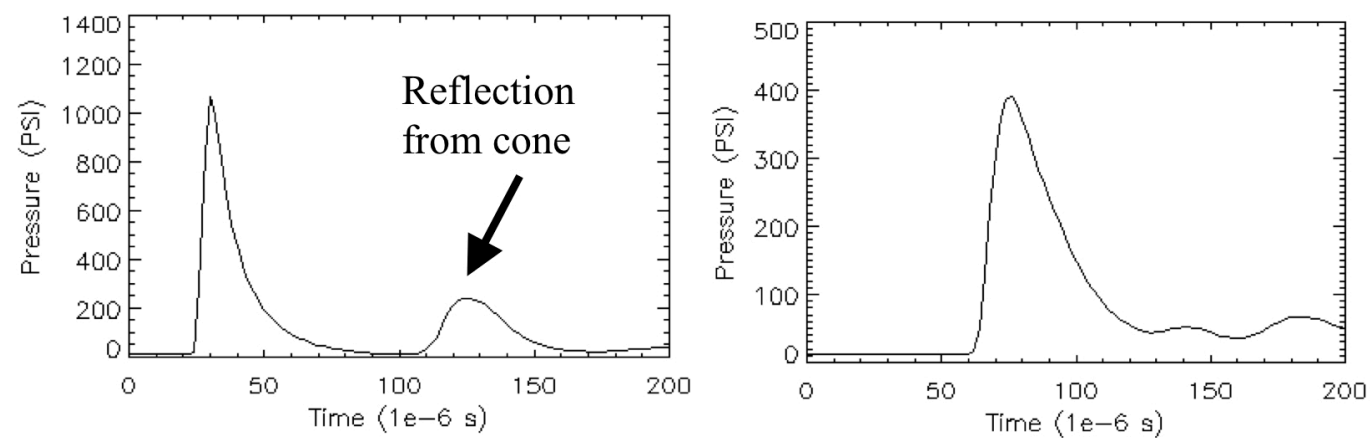

Figure XXXairsensor. Predicted dynamic overpressure profiles at airblast sensors. Left: Averaged pressure on sensor disc at near sensor (10 cm radius from source). Right Averaged pressure on sensor disc at far sensor (15 cm radius from source).
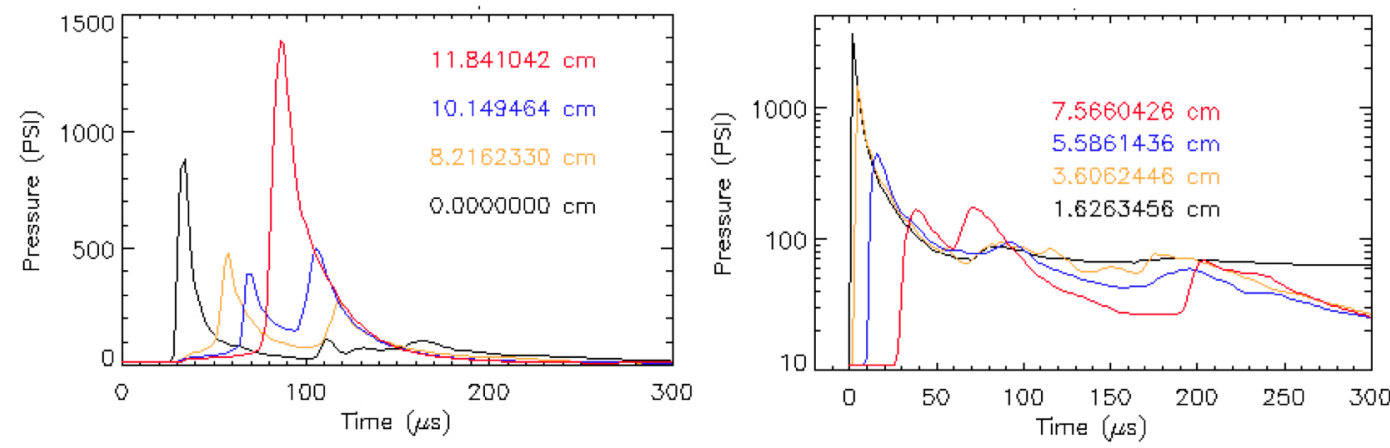

Figure XXXsf. Predicted dynamic overpressure profiles. Left: at various radii on cylinder boundaries. Right: at various heights on the large beam entry cone.
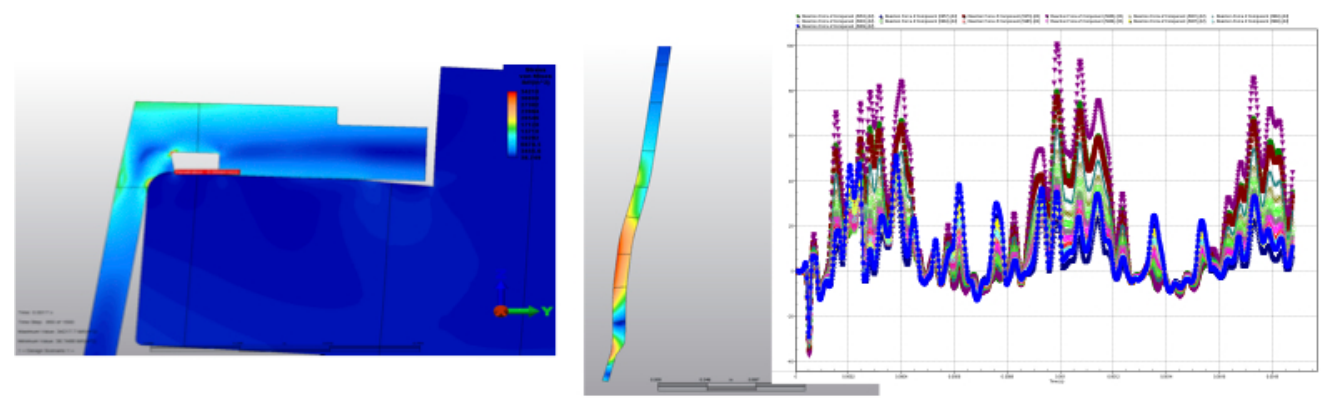

Figure XXXauto. Autodesk stress calculations. Left: Von Mises Stress at $1.7 \mathrm{~ms}$ \& near max stress of o-ring groove. Middle: VM Stress in cone wall. Right: Screw Z (tensile) forces. Max=100lb. Ave=50lb. 


\section{BK7 response}

Like fused silica, BK7 is amorphous glass and may exhibit a dispersive shock wave, in which case we would expect a ramp wave in the strain sensors embed in the BK7 (RRRdispersive).

Since an amorphous glass does not have a crystalline structure, it appears this dispersive behavior would not be due to a phase transition. Besides phase transitions, other discussion of dispersive shock waves in solids in the literature (RRRdispersive) concerns collapsing pores, deformation in components of layered materials, and large-amplitude rapid oscillations in the KdV equation producing an averaged dispersive wave, the application of which seems unclear in this case. The most probable mechanism for a dispersive shock wave appears to be a local densification of the atomic matrix.

Using HYDRA, we have produced the expected pressure history on the surface of the glass nearest the EPEC source. That history will be used to separately drive a block of BK7 glass in HYDRA. For this purpose, a material model for BK7 is being constructed, including equation of state and material strength properties. Currently we are expecting that densification will not occur at the pressures reached in the BK7 in the EPEC data experiment, except possibly near the source, meaning a ramp wave may not be seen at the sensors embedded in the BK7.

\section{Integrated modeling}

Integrated modeling that includes the atmosphere and realistic EOS and strength models for the solids is being developed. A significant challenge in this modeling is meshing atmosphere around the solid objects, especially around the beam entry cone, in a manner that captures the few- $\mu \mathrm{m}$ small displacements over most of the solid surfaces and allows the atmosphere to move past those surfaces.

RRRAutodesk

http://usa. autodesk.com/adsk/servlet/pc/index?sitelD=123112\&id=13773836

RRRdispersive A. Z. Zhuk, G. I. Kanel and A. A. Lash, Journal De Physique IV Colloque C8, supplement au Journal de Physique III (4), C8-403 (Sept. 1994); I. J. Fritz, J. Appl. Phys. 49(8) (August 1978);

RRRHYDRA Marinak, M. M., Kerbel, G. D., Gentile, N. A., Jones, O., Munro, D., Pollaine, S., Dittrich, T. R., Haan, S. W., "Three-dimensional HYDRA simulations of National Ignition Facility targets", Physics of Plasmas 
$8(5), 2275(2001)$.

RRRDANTE E. L. Dewald, K. M. Campbell, R. E. Turner, J. P. Holder, O. L. Landen, S. H. Glenzer, R. L. Kauffman, L. J. Suter, M. Landon, M. Rhodes, and D. Lee, "Dante soft X-ray power diagnostic for National Ignition Facility”, Rev. Sci. Instrum. 75, 3759 (2004).

RRRALE-AMR R. W. Anderson, N. S. Elliott and R. B. Pember, "An arbitrary Lagrangian-Eulerian method with adaptive mesh refinement for the solution of the Euler equations", Journal of Computational Physics 199 (2), 20 September 2004, Pages 598617.

RRRDYNA3D Energy \& Technology Review, September-October, 1993, pp. 1-5 